\title{
1 Collateral cleavage of 28s rRNA by RfxCas13d causes death of mice
}

\begin{tabular}{|c|}
\hline 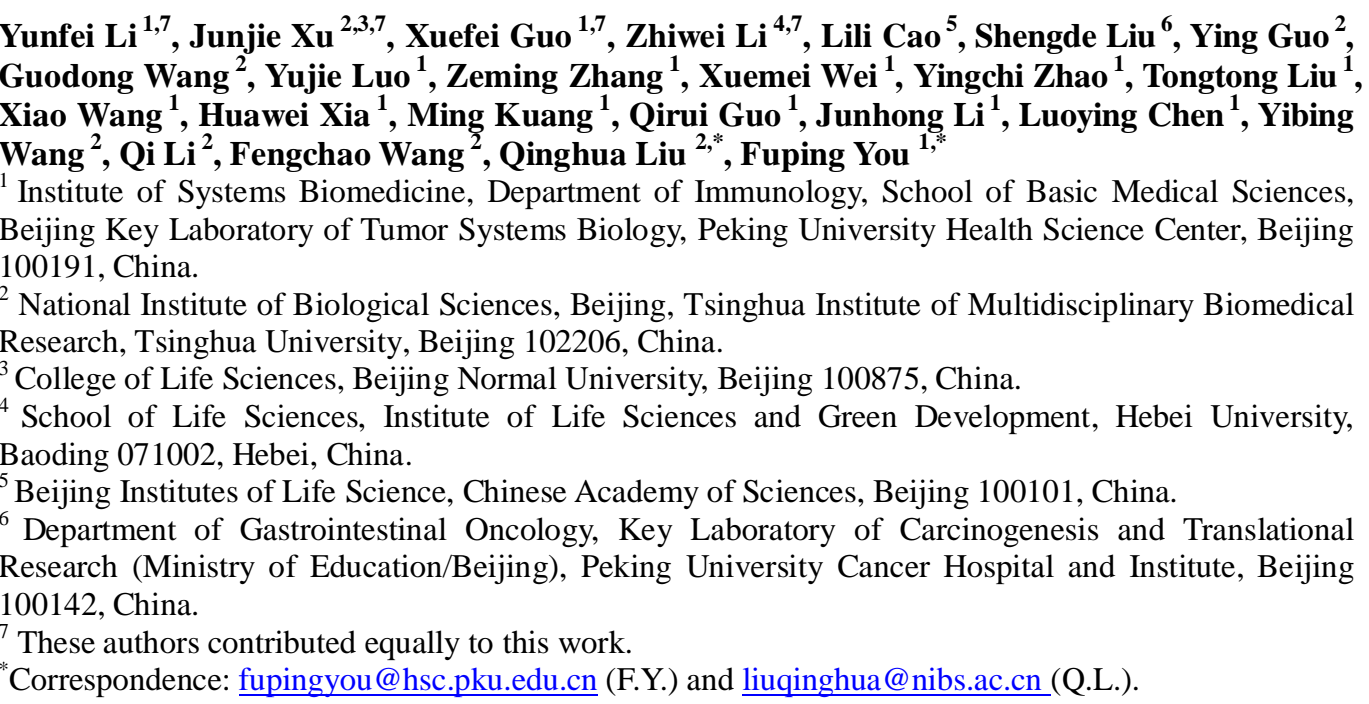 \\
\hline
\end{tabular}




\section{Summary}

21 The CRISPR-Cas13 system is an RNA-guided RNA-targeting system, and has been widely used in transcriptome engineering with potentially important clinical applications. However, it is still controversial whether Cas13 exhibits collateral activity in mammalian cells. Here, we found that knocking down gene expression using RfxCas13d in the adult brain neurons caused death of mice, which was not resulted from the loss of target gene function or off-target effects. Mechanistically, we showed that RfxCas13d exhibited collateral activity in mammalian cells, which is positively correlated with the abundance of target RNA. The collateral activity of RfxCas13d could cleave $28 \mathrm{~s}$ rRNA into two fragments, leading to translation attenuation and activation of the ZAK $\alpha$-JNK/p38-immediate early gene (IEG) pathway. These results provide new mechanistic insights into the collateral activity of RfxCas13d and warn that the biosafety of CRISPR-Cas13 system needs further evaluation before applying it to clinical treatments.

\section{Keywords}

RfxCas13d, collateral activity, death of mice, 28s rRNA breakage 


\section{Introduction}

Clustered regularly interspaced short-palindromic repeats (CRISPR) and accompanying CRISPR-associated (Cas) proteins constitute the adaptive CRISPR-Cas immune system in bacteria and archaea, which protects the bacteria from invaders, including phages and mobile genetic elements. The defense process can be divided into three stages: Adaptation, incorporation of foreign DNA fragments into CRISPR array as spacers; CRISPR RNA (crRNA) biogenesis, CRISPR array is transcribed into a long precursor crRNA (pre-crRNA), and then processed into mature crRNAs; Interference, Cas effector proteins, under the guidance of crRNAs, specifically recognize and cleave foreign genetic elements. The rapid evolutionary arms race between bacteria and mobile genetic elements has greatly enriched CRISPR-Cas systems, which have been harnessed for various research and therapeutic applications. According to the structure and function of Cas effector proteins, CRISPR-Cas systems can be categorized into two classes, which are further subdivided into six types (type $\square$-VI). Class 1 effectors comprise of multiple subunits, including type $\square, \square$, and $\square$, while class 2 effectors are single large proteins, including type $\square$, $\square$ and $\square^{[1]}$. Due to their simplicity, Class 2 CRISPR-Cas systems have been widely developed as genome editing and transcriptional regulating tools, such as DNA-targeting Cas9 and Cas12, RNA-targeting Cas13.

Cas 13 was originally found by mining microbial genome sequencing data using the highly conserved adaption protein Cas 1 as the anchor ${ }^{[2]}$. Protein sequence alignments revealed that Cas13 contains two higher eukaryotes and prokaryotes nucleotide-binding (HEPN) domains and is predicted to possess ribonuclease (RNase) activity ${ }^{[2]}$. It was confirmed by subsequent experiments that Cas13-crRNA complex recognizes and cleaves the target RNA via base pairing between the crRNA and the target RNA ${ }^{[3]}$. Surprisingly, binding of the target RNA to Cas13-crRNA complex also activates a nonspecific RNase activity, which promiscuously cleaves bystander RNAs without complementarity to the crRNA, leading to cell death or dormancy in bacteria ${ }^{[3,4]}$. This activity was referred as collateral activity of Cas 13 and had been ingeniously developed as molecular diagnosis tool in vitro ${ }^{[5]}$. However, this collateral activity has not been detected in mammals. Theoretically, compared with Cas9-mediated gene knockout technology, Cas13 can accurately distinguish different transcripts of the same gene, and then study their function individually. Besides, Cas13-mediated gene silencing does not change genomic DNA, so this gene silencing is reversible and considered safer than Cas9, which has advantages over Cas9 in the treatment of some acquired diseases. Moreover, accumulating evidence over the past decade highlights that noncoding RNAs play important roles in various cellular processes. Cas13 is more suitable for noncoding RNAs research than Cas9.

Currently, there are six subtypes identified in the Cas 13 family, including Cas $13 a$, Cas13b, Cas13c, Cas13d, Cas13X and Cas13 $\mathrm{Y}^{[2,6-10]}$. Since their discovery, Cas13's subtypes, such as LwaCas13a, PspCas13b, RfxCas13d and Cas13X.1, have been widely used in knockdown experiments in mammalian cells, exhibiting higher efficiency and specificity than traditional RNA interference, and no collateral activity was detected ${ }^{[3,6,8,10]}$. Among these Cas13's subtypes, due to its advantages in efficiency and size, RfxCas $13 \mathrm{~d}$ was applied in mammals via adeno-associated virus (AAV) delivery, with no side-effects reported ${ }^{[1-13]}$. But several research groups have different opinions about collateral activity of Cas13. Kang group first reported that the collateral activity of LwaCas13a occurred in U87 cells, non-specifically cleaving non-target RNAs, leading to cell death ${ }^{[14]}$. Later, they and collaborators reported that this phenomenon also existed in HepG2, AT2, B16F10 and GL261 cells ${ }^{[15,16]}$. Following their work, Gootenberg and Abudayyeh group reported that collateral activity was detected in U87 cells for LwaCas13a, PspCas13b and RfxCas13d, and in HepG2 and mES cells for RfxCas13d ${ }^{[17]}$. Yang group claimed that LwaCas13a, RfxCas13d and Cas13X.1 exhibited collateral activity when targeting transiently overexpressing mCherry, but not endogenous genes in $293 \mathrm{~T}$ cells ${ }^{[10]}$. Whereas, these studies did not figure out what effect collateral activity of Cas 13 has on mammalian cells. Therefore, it is still controversial whether collateral activity of Cas13 exists in mammalian cells. More importantly, the safety of applying Cas 13 to treatment needs to be carefully evaluated in animal models.

Here, we found that mice died when using RfxCas $13 \mathrm{~d}$ to knock down genes in brain neurons. The death would occur when target genes were present and obviously knocked down, but was not due to loss of gene function or off-target effects, which narrows down to collateral activity of Cas13. Then, we proved that RfxCas13d exhibited collateral activity in mammalian cells, which is 
92 positively correlated with the abundance of target RNA. The collateral activity of RfxCas13d 93 cleaved 28 s rRNA into two fragments, leading to translation attenuation and activation of the ZAK $94 \quad$ a-JNK/p38-IEG pathway.

95 
Results

Mice died when knocking down Sik3-S in neurons using RfxCas13d.

A recent study identified a Sleepy $\left(S i k 3^{S l p /+}\right.$ ) mouse strain, which carries a mutation in the gene encoding salt-inducible kinase 3 (SIK3), a member of the AMP-activated protein kinase (AMPK) family ${ }^{[18]}$. The Sleepy $\left(\mathrm{Sik}^{\mathrm{Slp/+}}\right)$ mice exhibit over $4 \mathrm{~h}$ increase in daily non-rapid eye movement sleep (NREMS) time and constitutively elevated NREMS delta power relative to wild-type (WT) littermates ${ }^{[18]}$. Interestingly, the $S i k 3$ gene encodes multiple transcripts due to alternative splicing. Our recent study identified a new Sik3-S transcript encoding $\sim 72 \mathrm{kDa}$ short isoform of SIK $3^{[19]}$ (Fig. S1a). To investigate the role of SIK3-S in sleep regulation, RfxCas13d was leveraged to specifically knock down $S i k 3-S$ by taking advantage of its ability to distinguish different transcripts of same gene (Fig. 1a). We designed eight crRNAs targeting Sik3-S and examined their knockdown efficiency in N2a cells through RT-qPCR (Fig. S1b). Our results showed that, in collaboration with RfxCas13d, all eight crRNAs, especially crRNA 1 and 8, caused significant knockdown of Sik3-S expression (Fig. 1b). Transcriptome analysis revealed that Sik3 was specifically down-regulated while almost all the other genes remained unchanged when RfxCas13d was co-transfected with Sik3-S crRNA 1 or 8, compared with non-targeting (NT) crRNA (Fig. 1c).

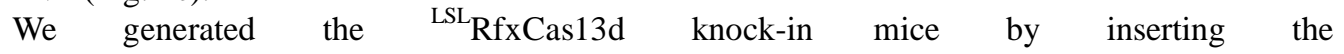
CAG-loxP-STOP-loxP-RfxCas13d cassette into the Rosa26 locus by homologous recombination (Fig. S1c). To knock down Sik3-S expression in the adult brain neurons, we retro-orbitally injected

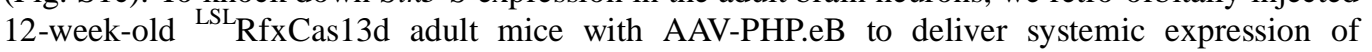
U6-driven crRNA and hSYN-driven Cre (Fig. S1d). AAV-PHP.eB could efficiently cross the blood brain barrier and transduced the majority of neurons and astrocytes across the adult mouse brain ${ }^{[20]}$. The human synapsin 1 gene promoter (hSYN) restricted the expression of Cre recombinase in neurons. Subsequently, Cre recombinase mediated excision of a tripartite transcriptional stop cassette (STOP) flanked by loxP to release the expression of RfxCas13d. RfxCas13d, under the guidance of Sik3-S crRNAs, specifically recognized and cleaved Sik3-S transcripts (Fig. 1d). Unexpectedly, mice injected with AAV-PHP.eB containing $S i k 3-S$ crRNA 8 began to lose weight at $\sim 20$ days post injection (dpi), and died at $\sim 24$ dpi (Fig. 1e-f). Brain lysates from Sik3-S crRNA 8 group, but not NT crRNA or Sik3-S crRNA 1 group, showed decreased SIK3-S expression, demonstrating that Sik3-S expression was specifically knocked down in this group (Fig. 1g). This phenomenon prevented our experiments from continuing, but aroused our curiosity-why mice died when knocking down Sik3-S using RfxCas13d in the adult mouse brain neurons. 
Several previous studies have proved that RfxCas $13 \mathrm{~d}$ can be used to knock down endogenous genes in vivo with no reported side-effects in liver, brain and eyes ${ }^{[11-13]}$. In addition, although the homozygous Sik3 knockout mice can be created, but exhibit impaired chondrocyte during development, neonatal lethality and reduced size, indicating that Sik3 is essential for mouse health and survival ${ }^{[21,22]}$. Therefore, we first guessed whether the death of mice was caused by down-regulation of Sik3-S. To test this, we knocked out Sik3 in the same neurons of mice by conventional Cre-loxP system. We generated Sik3-E5 ${ }^{\text {flox }}$ mice by inserting two loxP sites into both sides of exon 5 in $S i k 3$ gene locus, and then delivered AAV-PHP.eB carrying hSYN-driven Cre into Sik3-E5 ${ }^{\text {flox }}$ mice to knock out Sik3, with WT mice as control (Fig. S2a). At 21 dpi, brain lysates from Sik3-E5 ${ }^{\text {flox }}$ mice showed lower SIK3-S expression level compared to WT mice (Fig. 2a). But Sik3-E5 ${ }^{\text {flox }}$ mice behaved as normal as WT mice without loss of body weight or death (Fig. 2b-c), which means that knocking out Sik3 in neurons will not cause mouse death. Thus, the mouse lethality that occurred when using RfxCas13d to knock down Sik3-S expression had nothing to do with the loss of functional SIK3-S.

Map2, Tau and NeuN were well characterized neuron marker genes. Homozygous knockout of each of these genes did not lead to death of mice ${ }^{[23-25]}$. We thus selected them as targets and knocked down each of three targets in the same way as knocking down Sik3-S in vivo. In theory, when knocking down these genes individually using RfxCas $13 \mathrm{~d}$ in adult mouse brain neurons, the mice will not die due to the loss of these genes. We designed six crRNAs for each gene and tested their knockdown efficiency by RT-qPCR (Fig. 2d-e). Since N2a cells do not express NeuN, we tested the efficiency of crRNAs by knocking down expression of co-transfected NeuN plasmid in HEK293T (Fig. 2f). The two best crRNAs for each gene were selected for follow-up experiments. Transcriptome analysis showed that these three genes were specifically knocked down using corresponding crRNAs in tandem with RfxCas13d (Fig. 2g-i). Following the same protocol of knocking down Sik3-S in vivo, we knocked down these three genes respectively in ${ }^{\mathrm{LSL}} \mathrm{RfxCas} 13 \mathrm{~d}$ mice. Results showed that mice in Map2 crRNA 3, Mapt crRNA 6 and Rbfox3 crRNA 5 groups showed significant loss of body weight and death, while mice in the other groups behaved normally and survived (Fig. 2j-o). Besides, brain lysates showed that these three target genes were down-regulated in corresponding death groups (Fig. 2p-r). Taken together, these data suggested that RfxCas13d mediated mouse death was not due to the loss of target gene function. 
RfxCas13d mediated lethality was not caused by off-target effects.

One ongoing concern using any CRISPR-Cas system for gene editing is off-target effects ${ }^{[26]}$. Thus, it's necessary to determine whether RfxCas13d-mediated mouse death is caused by off-target effects. Firstly, Ai14 (Rosa-CAG-LSL-tdTomato-WPRE) reporter mice were introduced and crossed with ${ }^{\text {LSL }}$ RfxCas $13 \mathrm{~d}$ mice to generate ${ }^{\mathrm{LSL}} \mathrm{RfxCas} 13 \mathrm{~d}^{+/ f 1} \mathrm{Ai} 14^{+/ f 1}$ mice ${ }^{[27]}$ (Fig. S3a-b). We designed seven crRNAs targeting tdTomato and tested their knockdown efficiency in N2a cells stabling expressing tdTomato by RT-qPCR. Among these crRNAs, crRNA 4 and 7 significantly damped the expression of tdTomato (Fig. 3a). Transcriptome analysis showed that tdTomato was specifically knocked down in cells transfected with tdTomato crRNA 4 or 7 (Fig. 3b). Moreover, the expression of the reported lethal genes was not affected by these two crRNAs. Then, we knocked down tdTomato in ${ }^{\mathrm{LSL}} \mathrm{RfxCas} 13 \mathrm{~d}^{+/ f 1} \mathrm{Ai} 14^{+/ f 1}$ mice in the same way as knocking down Sik3-S. Results showed that mice injected with AAV-PHP.eB carrying tdTomato crRNA 7 began to lose weight at $\sim 12 \mathrm{dpi}$ and died at $\sim 15 \mathrm{dpi}$, mice in the other groups behaved normally and survived (Fig. 3c-d). Brain lysates showed that tdTomato expression was lower in tdTomato crRNA 7 group than NT crRNA or tdTomato crRNA 4 group (Fig. 3e).

Next, we simultaneously injected AAV-PHP.eB carrying tdTomato crRNA 4, 7 or NT crRNA into ${ }^{\mathrm{LSL}} \mathrm{RfxCas} 13 \mathrm{~d}$ and ${ }^{\mathrm{LSL}} \mathrm{RfxCas} 13 \mathrm{~d}^{+/ f 1} \mathrm{Ai} 14^{+/ f l}$ mice. Theoretically, if $\mathrm{RfxCas} 13 \mathrm{~d}$-mediated mouse death was caused by off-target effects, both ${ }^{\mathrm{LSL}} \mathrm{RfxCas} 13 \mathrm{~d}$ and ${ }^{\mathrm{LSL}}{ }_{\mathrm{Rfx} C a s} 13 \mathrm{~d}^{+/ f 1} \mathrm{Ai} 14^{+/ f l}$ mice injected with AAV-PHP.eB carrying tdTomato crRNA 7 would die. However, if RfxCas13d-mediated mouse death was not caused by off-target effects, only ${ }^{\mathrm{LSL}}{ }_{\mathrm{Rfx}} \mathrm{Cas} 13 \mathrm{~d}^{+/ f 1} \mathrm{Ai} 14^{+/ f l}$ mice injected with AAV-PHP.eB carrying tdTomato crRNA 7 would die (Fig. 3f). Results showed that only ${ }^{\mathrm{LSL}} \mathrm{RfxCas} 13 \mathrm{~d}^{+/ \mathrm{fl}} \mathrm{Ai} 14^{+/ \mathrm{fl}}$ mice injected with AAV-PHP.eB carrying tdTomato crRNA 7 began to lose body weight at $\sim 12$ dpi and died at $\sim 15 \mathrm{dpi}$, mice in the other groups behaved normally (Fig. 3g-h). These data suggested that RfxCas13d-mediated mouse death was not caused by off-target effects. Moreover, since tdTomato is a foreign gene and has no function in brain neurons, this result further support that RfxCas13d mediated lethality had nothing to do with the loss of target gene function. 
The collateral activity of RfXCas13d was determined by the abundance of target RNA in mammalian cells.

Above data ruled out the possibility that the death of mice was caused by the loss of target gene function or off-target effects. In addition, interestingly, only when the target genes were present and obviously knocked down, the mice would die. It was reminiscent of collateral activity of Cas13. Firstly, we verified findings in previous studies ${ }^{[14,17]}$. We transfected in vitro-synthesized EGFP crRNA or NT crRNA into U87 cells stably expressing LwaCas13a and EGFP. The protein sequence of LwaCas13a and the sequence of crRNA we used are the same as previously reported $^{[14]}$. Results showed that EGFP mRNA was significantly knocked down at $4 \mathrm{~h}$ and $8 \mathrm{~h}$ post transfection of EGFP crRNA, not NT crRNA (Fig. S4a). RNA denaturing gel electrophoresis of total RNA showed that $28 \mathrm{~s}$ and $18 \mathrm{~s}$ rRNAs were intact, and not cleaved into multiple bands as previous reported (Fig. S4b). Gootenberg and Abudayyeh group reported that LwaCas13a, PspCas13b and RfxCas13d exhibit collateral activity in U87 cells, thereby reducing cell viability ${ }^{[17]}$. But according to their results, transfection of plasmids encoding LwaCas13a, PspCas13b or RfxCas13d into U87 cells, regardless of with targeting or NT crRNA, would affect cell viability, indicating that cell viability changes have nothing to do with collateral activity. Therefore, it is still unclear whether collateral activity of Cas 13 exists in mammalian cells.

We transiently transfected plasmids encoding RfxCas13d, tdTomato and crRNAs into HEK293T cells. Results showed that the protein and RNA level of RfxCas13d decreased, when it knocked down tdTomato under guidance of tdTomato crRNAs not NT crRNA (Fig. 4a-b). However, this phenomenon would not occur when there was no target gene expression or using catalytically dead RfxCas13d (dRfxCas13d) (Fig. 4a-b). This suggested that the collateral activity of RfxCas13d was activated to cleave its own mRNA when RfxCas13d-crRNA complex bound and cleaved tdTomato mRNA. Interestingly, changes in protein levels were more obvious than changes in RNA levels (this will be explained later). The same phenomenon was observed when knocking down Sik3-S (Fig. S4c-d). LwaCas13a and PspCas13b also exhibited similar characteristics (Fig. S4e-f). Yang group found that LwaCas13a, RfxCas13d and Cas13X.1 exhibited collateral activity when targeting transiently overexpressing mCherry, but not endogenous genes, using EGFP stably expressed in HEK293T as the indicator of collateral effects ${ }^{[10]}$, which gives us a hint that collateral activity of Cas13 may relates with the abundance of target RNA. Besides, in bacteria, Cas13-induced dormancy requires target RNA levels to exceed an expression threshold ${ }^{[28]}$. And in vitro experiments proved that collateral activity of Cas13 is positively correlated with the abundance of target $\mathrm{RNA}^{[5,29]}$. To verify whether this correlation is also present in mammalian cells, a HEK293T cell line inducibly expressing tdTomato was constructed leveraging the tetracycline-controlled Tet-On inducible expression system, and then transfected with plasmids encoding RfxCas13d and crRNAs under different concentration doxycycline treatment (Fig. 4c). Results showed that RfxCas13d was negatively correlated with tdTomato at the expression level, under co-transfection of RfxCas13d and tdTomato crRNAs instead of NT crRNA (Fig. 4c). These data indicated that the collateral activity of RfxCas13d was triggered and positively correlated with the abundance of target RNA in mammalian cells, when targeting exogenous genes.

Next, we determined whether the collateral activity of RfxCas13d occurs when targeting endogenous genes in mammalian cells. We noticed that these endogenous genes previously used as targets are low in abundance ${ }^{[8,10]}$. It is possible that collateral activity had been activated, but it was too weak to be detected. Therefore, we here selected several highly expressed genes as targets and designed four crRNAs for each gene. Then, plasmids encoding RfxCas13d/dRfxCas $13 \mathrm{~d}$ and crRNAs were transiently transfected into HEK293T cells. The knockdown efficiency of crRNAs was measured by RT-qPCR (Fig. 4d-f and S4g-j), and collateral activity was detected by measuring RfxCas13d expression level (Fig. 4g-i and S4k-n). Results showed that RfxCas13d, not dRfxCas13d, was down-regulated when targeting highly expressed genes, indicating that collateral activity was activated (crRNAs pointed by red arrows in Fig. 4g-i and S4k-n). Taken together, these data demonstrated that RfxCas13d exhibited collateral activity in mammalian cells, which is positively correlated with the abundance of target RNA. 
The collateral activity of RfxCas13d cleaves 28s rRNA into two fragments, leading to translation attenuation and activation of $\mathrm{ZAK} \alpha$-JNK/p38-IEG pathway.

Although it was confirmed that the collateral activity of RfxCas13d existed in mammalian cells. It remains unknown whether and how this activity affects the biological process of cells. To this end, we constructed a HEK293T cell line stably expressing RfxCas13d (HEK293T-RfxCas13d) and then transfected with plasmids encoding target gene and corresponding crRNAs (Fig. S5a). In this way, RfxCas13d was fully expressed before the collateral activity was induced, which prevents the collateral activity of RfxCas $13 \mathrm{~d}$ from affecting its own expression by cleaving RfxCas13d mRNA (Fig. S5b). Thus, more RfxCas13d protein and the secondary induced collateral activity could be preserved than co-transfection. Then, cells were harvested $24 \mathrm{~h}$ post transfection for cell cycle distribution analysis, total RNA integrity analysis and RNA-seq (Fig. S5a). Analysis of total RNA integrity showed that, not only 28 s and 18s rRNA, two additional bands but also were detected when co-transfecting of target genes and corresponding targeting crRNAs instead of NT crRNA into HEK293T-RfxCas13d cells (Fig. 5a). The same phenomenon occurred when targeting endogenous highly expression genes (Fig. S5c). In terms of size, these two additional bands looked like the products of $28 \mathrm{~s}$ rRNA being cleaved. To test this, we did oligonucleotide extension assay to map cleavage sites (Fig. S5d). PCR and sanger sequencing revealed that $28 \mathrm{~s}$ rRNA was cut into two fragments, one fragment of $\sim 2100 \mathrm{nt}$ and the other segment of $\sim 2800 \mathrm{nt}$ (Fig. S5e-f). Noticeably, several sequencing results detected until $\sim 2187 \mathrm{nt}$ of $28 \mathrm{~s}$ rRNA (marked by blue color), and one sequencing result revealed that a poly-A tail was added to $2187 \mathrm{nt}$ of $28 \mathrm{~s}$ rRNA (marked by brown color) (Fig. S5f). There is "UU" behind $2187 \mathrm{nt}$ of the complete $28 \mathrm{~s}$ rRNA (marked by red color) (Fig. S5f). And we proved that the collateral activity of RfxCas13d prefers to cleave poly-U in vitro, which is consistent with previous study ${ }^{[30]}$ (Fig. S5g-h). Therefore, this "UU" site (2188-2189nt) is likely to be the cleavage site by RfxCas13d on 28 s rRNA (Fig. 5b). Those slightly shorter fragments may be caused by post-cleavage degradation. Interestingly, why did the collateral activity of RfxCas13d cleave $28 \mathrm{~s}$ rRNA but not $18 \mathrm{~s}$ rRNA? Theoretically, the abundance of 18s rRNA is also high, and there are also "UU" sites on it that can be cleaved. Besides, why did the collateral activity of RfxCas13d cut 28 s rRNA at this "UU" site not others? We speculated that it may be due to the structure of RNA and RNA binding proteins (RBPs) that protect rRNAs from being cut. To test our speculation, we extracted total RNA from HEK293T cells and reconstituted the collateral activity of RfxCas13d in vitro, and founded that $28 \mathrm{~s}$ and $18 \mathrm{~s}$ rRNA were cleaved into multiple fragments (Fig. S5i), indicating that RNA structure and RBPs were involved in protecting RNA from the collateral activity of RfxCas13d. 28s rRNA is an important component of the ribosome. To determine whether the translation function of the ribosome was affected due to $28 \mathrm{~s}$ rRNA breakage, SUnSET assay was employed to monitor protein synthesis. To avoid the impact of SIK3-S enzyme activity on translation, we used kinase dead SIK3-S (SIK3-S-K37M) instead of WT SIK3-S (Fig. S6a). Results showed that protein synthesis was attenuated when target genes were co-transfected with targeting crRNA, not NT crRNA (Fig. 5c). These data suggested that the collateral activity of RfxCas13d cleaved 28s rRNA into two fragments, thereby affecting the translation function of the ribosome. This may explain why in Fig. 4a, changes in protein levels of RfxCas13d were more obvious than changes in RNA levels (Fig. S5b). Cell cycle distribution analysis showed that co-transfection of target gene and targeting crRNAs, but not NT crRNA leaded to cell cycle arrest at G1 phase (Fig. 5d and S6b). This may be caused by impaired translation of protein.

RNA-seq analysis showed that there were 509 common differentially expressed genes from four sets of comparisons (Fig. 5e). Interestingly, these were all up-regulated genes compared with NT crRNA (Supplementary Table 1). Among these genes, we noticed that multiple genes with obvious difference belong to IEGs (Fig. 5f). Besides, transcription factor enrichment analysis of 509 genes showed that multiple enriched transcription factors mediate the expression of IEGs, including JUNB, FOSB, JUN, EGR1, EGR2, ATF3, NR4A3, NR4A1 and CSRNP1 (Fig. 5g). IEGs are genes which are activated transiently and rapidly in response to various cellular stimuli. There are several pathways that lead to the activation of IEGs, such as the RhoA-actin and the ERK, JNK and p38 MAPK pathways ${ }^{[31]}$. We used inhibitors of these pathways to block IEGs expression, and found that IEGs expression can be blocked by p38 and JNK inhibitors not MEK1/2 or RhoA/C inhibitors (Fig. S6c-d). The combination of p38 and JNK inhibitors worked better (Fig. S6c-d). Consistently, western blot revealed increased phosphorylation of p38 and JNK, but not ERK1/2 (Fig. 5h), demonstrating that JNK and p38 were responsible for the expression of IEGs. Previous 
studies reported that ZAK $\alpha$, the long isoform of ZAK, senses ribotoxic stress caused by rRNA damage or ribosome impairment, and then activates $\mathrm{p} 38$ and JNK pathways ${ }^{[32]}$. We speculated that ZAK $\alpha$ may sense 28 s rRNA breakage caused by RfxCas $13 \mathrm{~d}$ and activate JNK and p38 pathways. To test this, we firstly used two ZAK inhibitors $\left(6 \mathrm{p}^{[33]}\right.$ and HY180) to block IEGs expression. IEGs expression can be inhibited by both inhibitors in a dose-dependent manner (Fig. S6e-f). Then, we knocked out ZAK in HEK293T-RfxCas13d cells and found that IEGs expression was blocked in ZAK knockout cells not PKR (another ribotoxic stress sensor) knockout cells (Fig. 5i-k). Besides, re-expression of ZAK $\alpha$ not ZAK $\beta$ (the short isoform of ZAK) in ZAK knockout cells can rescue the expression of IEGs (Fig. 5i-k). Consistently, western blot demonstrated that phosphorylation of p38 and JNK was blocked in ZAK knockout cell, and can be rescued by re-expression of ZAK $\alpha$ not ZAK $\beta$ (Fig. 51). These data proved that ZAK $\alpha$ sensed $28 \mathrm{~s}$ rRNA breakage caused by RfxCas13d and mediated phosphorylation of p38 and JNK, then activating IEGs expression. Taken together, these data demonstrated that the collateral activity of RfxCas $13 \mathrm{~d}$ cleaves $28 \mathrm{~s}$ rRNA into two fragments, leading to translation attenuation and activation of the 315 ZAK $\alpha$-JNK/p38-IEG pathway (Fig. 5m). 


\section{Discussion}

318 Here, we initially utilized RfxCas13d to specifically knock down Sik3-S in the adult mouse brain neurons for studying its role in sleep. Unexpectedly, mice died when SIK3-S was obviously knocked down, which arises our curiosity about the death of mice. Subsequent in vivo experiments ruled out the possibility that RfxCas13d-mediated mouse death was due to loss of gene function or off-target effects, and demonstrated that mice would die when target genes were present and obviously knocked down. These data reminded us whether the death of mice was caused by activation of collateral activity when RfxCas13d recognized and cleaved target genes. To prove this, we confirmed that RfxCas13d exhibits collateral activity in mammalian cells, which is related to the abundance of target genes. Then, we founded that the collateral activity of RfxCas $13 \mathrm{~d}$ cleaves 28s rRNA into two fragments, leading to translation attenuation and activation of ZAKa -JNK/p38-IEG pathway. In conclusion, we found that RfxCas13d exhibits collateral effects in mammalian cells, causing death in mice.

Previous studies used RfxCas13d to knock down endogenous genes in vivo, such as in brain glia, liver and eyes, without side-effect reported. But we observed mouse death when using RfxCas13d to knock down endogenous genes in brain neurons. This discrepancy in experimental outcome may be because neurons are more important than other kinds of cells and not easy to regenerate, so neurons are more sensitive to collateral activity, and animal's performance is more obvious. The death of mice warned us that the safety of RfxCas13d needs to carefully evaluation in animal models before applying it to treatment.

During the research, we found that exogenous genes were more likely to be cleaved by the collateral activity of RfxCas13d than endogenous genes, which may be due to the fact that endogenous genes hold more comprehensive RNA structure and closer combination with RBPs than exogenous genes. Therefore, changes in the expression of exogenous genes, such as EGFP or mCherry, are more suitable as indicators of the collateral activity of RfxCas $13 \mathrm{~d}$, but the cleavage of exogenous genes does not represent that endogenous genes will also be cleaved.

The cleavage of 28s rRNA was easily observed, due to its high abundance and important role in cells. But it is still unclear whether the collateral activity of RfxCas13d cuts other RNAs. We observed 509 common differentially expressed genes from four sets of comparisons. IEGs are only part of them. It is unclear why other genes were up-regulated, which may be the consequence of the collateral activity of RfxCas13d cleaving RNAs other than 28s rRNA. So, there is an urgent need to establish a method to detect all the cleavage sites of Cas13's collateral activity, which is crucial for Cas13's optimization in the future. When RfxCas13d recognizes and cleaves the target RNA, its collateral activity is activated to cleaves RfxCas13d mRNA and 28s rRNA, which in turn negatively regulates its own expression and collateral activity. Therefore, it is recommended to express Cas13 in cells in advance, and then to induce its collateral activity and study cleavage sites. Studying the cleavage mechanism of Cas13's collateral activity will not only direct Cas13's optimization in transcriptome engineering via reducing or removing collateral activity, but also inspire us to develop new applications in mammalian cells taking advantage of collateral activity. 
357 Acknowledgement

358 This work was supported by the National Key Research and Development Program of China 359 (2016YFA0500300; 2020YFA0707800), the National Natural Science Foundation of China 360 (31570891; 31872736; 32022028; 81991505), Peking University Clinical + X 361 (PKU2020LCXQ009), the Peking University Medicine Fund (PKU2020LCXQ009) and a grant from Zhuhai Science and Technology Innovation Bureau (ZH22036302200063PWC to Z. Yin). Thanks Yichen Deng for help in FCAS. Thanks Prof. Xiaoyun Lu for providing ZAK inhibitors $6 p$ and HY180.

\section{Author Contributions}

Y.L., J.X., F.Y. and Q.L. conceived the study and analyzed the data. Y.L., J.X. and Z.L. performed most of the experiments. X.G. was responsible for RNA-seq analysis. Y.W., S.L. and L.C. provided advise and technical help. Q.L. was responsible for AAV preparation. S.W. generated

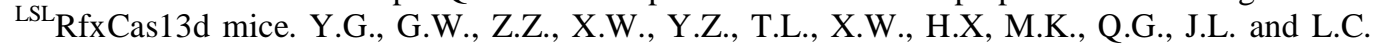
assisted in the molecular experiments. Y.L., F.Y. and Q.L. wrote and revised the paper. J.X, S.L. and L.C. helped with the paper revision.

\section{Competing Interests}

The authors have no conflicts of interest to declare 


\section{STAR国Methods}

\section{Cell culture}

HEK293T, N2a and U87 cells were obtained from ATCC. Cells were cultured in DMEM medium supplemented with $10 \%$ FBS (Gibco) and $100 \mathrm{U} / \mathrm{ml}$ Penicillin-Streptomycin in a humidified incubator at $37{ }^{\circ} \mathrm{C}$ with $5 \% \mathrm{CO}_{2}$. Additional $1 \%$ glutamine for U87 cells.

\section{Animals}

All animals care and use adhered to the Guide for the Care and Use of Laboratory Animals of the Chinese Association for Laboratory Animal Science. All procedures of animal handling were approved by the Animal Care Committee of Peking University Health Science Center (permit number LA 2016240). ${ }^{\text {LSL }}$ RfxCas13d and Sik3-E5 ${ }^{\text {llox }}$ mice on a C57BL/6J background were generated by the Transgenic Animal Center, NIBS, Beijing, China. Ai14 reporter mice were purchased from The Jackson Laboratory. Wild-type mice were purchased from Department of Laboratory Animal Science of Peking University Health Science Center, Beijing, China. Mice were kept and bred in pathogen-free conditions.

\section{Plasmids construction}

Plasmids used in this study were prepared by standard molecular biology techniques and coding sequences entirely verified. All the mutants were constructed by standard molecular biology technique. Each mutant was confirmed by sequencing.

\section{Reagents and antibodies}

Polyethylenimine (PEI) (764582, Sigma-Aldrich) and jetPRIME (114-15, Polyplus) were used for transfection. In vitro-synthesized crRNAs were purchased from GenScript. Quenched fluorescent reporter RNA was purchased from General Biology. Inhibitors used in this study including the following: p38 inhibitor SB203580 (HY-10256, MCE); JNK inhibitor SP600125 (HY-12041, MCE); MEK1/2 inhibitor U0126 (HY-12031, MCE); RhoA/C inhibitor (S7719, Selleck). ZAK inhibitors 6P and HY180 are gift from Prof. Xiaoyun Lu, Jinan University. Antibodies used in this study include the following: anti-HA (Rabbit, H6908, Sigma-Aldrich); anti-HA (Mouse, self-made); anti-SIK3 (Rabbit, self-made); anti- $\beta$-Tubulin (Mouse, HC101, TransGen Biotech); anti-ACTB (Mouse, 60008-1-Ig, Proteintech); anti-GAPDH (Mouse, 60004-1-Ig, Proteintech); anti-NeuN (Rabbit, 26975-1-AP, Proteintech); anti-Tau (Rabbit, 10274-1-AP, Proteintech); anti-MAP2 (Rabbit, 17490-1-AP, Proteintech); anti-ZAK (Rabbit, 28761-1-AP, Proteintech); anti-PKR (Rabbit, 18244-1-AP, Proteintech); anti-p-p38 (Rabbit, 4511, CST); anti-p-JNK (Rabbit, 4370, CST); anti-p-ERK1/2 (Rabbit, ET1609-42, HUABIO); HRP-conjugated Affinipure Goat Anti-Rabbit $\operatorname{IgG}(\mathrm{H}+\mathrm{L})$ (SA00001-2, Proteintech); HRP-conjugated Recombinant Rabbit Anti-Mouse IgG Kappa Light Chain (SA00001-1, Proteintech).

\section{AAV-PHP.eB packaging, purification and injection}

AAV-PHP.eB was packaged in AAVpro 293T cells (632273, Clontech). PHP.eB (Addgene\#103005), pHelper (240071-54, Agilent) and transfer plasmids were co-transfected to AAVpro $293 \mathrm{~T}$ cells by PEI MAX (24765, Polysciences). Cells were harvested by cell lifter (70-2180, Biologix) $72 \mathrm{~h}$ post-transfection. The cell pellets were suspended in 1x Gradient Buffer $(10 \mathrm{mM}$ Tris- $\mathrm{HCl} \mathrm{pH}=7.6,150 \mathrm{mM} \mathrm{NaCl}, 10 \mathrm{mM} \mathrm{MgCl}$ ). Five repeated cycles of liquid nitrogen freezing, $37^{\circ} \mathrm{C}$ water bath thawing and vortex were used to lyse cell. Then $\geq 50 \mathrm{U} / \mathrm{ml}$ of Benzonase nuclease (E1014, Milipore) were added to cell lysates and incubated at $37^{\circ} \mathrm{C}$ for $30 \mathrm{~min}$. Centrifuge the cell lysate at $21,130 \mathrm{~g}$ for $30 \mathrm{~min}$ at $4^{\circ} \mathrm{C}$ and transfer the supernatant to a pre-build iodixanol (D1556, Optiprep) step gradients $(15 \%, 25 \%, 40 \%$ and $58 \%)$ for ultracentrifugation purification. Vacuum centrifuge at $41,000 \mathrm{rpm}, 4^{\circ} \mathrm{C}$ for $4 \mathrm{~h}$, the virus particles were in the layer of 
$40 \%$ iodixanol gradient. Purified virus were extracted from the $40 \%$ virus containing layer by needle and concentrated using Amicon filters (UFC801096, EMD) and formulated in sterile phosphate-buffered saline (PBS) supplemented with 0.01\% Pluronic F68 (24040032, Gibco). Virus titers were determined by $\mathrm{qPCR}$ while a linearized AAV plasmid as a standard. $1 \times 10^{12} \mathrm{vg} /$ mouse AAV-PHP.eB were delivered into mice via retro-orbital injection.

\section{RNA extraction and reverse transcription quantitative real-time PCR (RT-qPCR)}

Total RNA from cells were isolated using TRIzol reagent (DP424, TIANGEN). 1ug RNA was reverse transcribed using HiScript II Q RT SuperMix (R223-01, Vazyme). Levels of these indicated genes were analyzed by qPCR amplified using SYBR Green (Q311, Vazyme). Data shown are the relative abundance of the indicated mRNA normalized to ACTB or GAPDH. The primers were list in Table 1.

\section{Measurement of crRNAs' knockdown efficiency}

Plasmids encoding RfxCas13d (addgene\#109049) and crRNAs (addgene\#109053) were transfected into N2a cells. $48 \mathrm{~h}$ after transfection, GFP-positive cells were sorted and collected through Fluorescence-Activated Cell Sorting (FACS), and then were extracted for total RNA. Then, levels of indicated genes were measured by RT-qPCR. All crRNAs used in this paper were listed in Table 2.

\section{Cell cycle distribution analysis}

Cells were washed and collected using PBS to get rid of serum proteins at centrifugation at 1,200 rpm, $5 \mathrm{~min}$. Resuspend pellet using precooling 70\% EtOH solution to fix cells at least 30 $\min$ at $4^{\circ} \mathrm{C}$. The cells can remain in this solution for up to one week. Dilute EtOH/cell suspension with PBS. Spin at 2,000-2,200 rpm for 10 min spin. Cells are much harder to pellet in EtOH. If $\mathrm{EtOH}$ is not diluted and the increased rate is not used, significant cell loss will be noticed. Wash cells three times using PBS and then stain cells using DAPI staining solution (C1005, Beyptime) for $30 \mathrm{~min}$. Finally, cells were recorded by Fluorescence Activated Cell Sorting (FACS) and analyzed by FlowJo.

\section{Western Blotting.}

Cells were washed with PBS and lysed by incubation on ice for 10 min with RIPA lysis buffer ( $50 \mathrm{mM}$ Tris, $150 \mathrm{mM} \mathrm{NaCl}, 0.1 \%$ SDS, $0.5 \%$ sodium deoxycholate, $1 \%$ Triton X-100, protease cocktail [C0001, Targetmol], and $1 \mathrm{mM}$ PMSF). Brain tissue was firstly grinded in a mortar cooled on liquid nitrogen, and then lysed by incubation on ice for 30 min with RIPA lysis buffer. Supernatants were collected by centrifugation at $12,000 \mathrm{rpm}$ for $10 \mathrm{~min}$ at $4{ }^{\circ} \mathrm{C}$, and then mixed up with loading buffer and boiled for $10 \mathrm{~min}$. Samples were resolved by SDS/PAGE and transferred to 0.22 um nitrocellulose membrane (P-N66485, Pall). The membrane was blocked using skim milk for $30 \mathrm{~min}$, then incubated overnight with primary antibodies, further incubated with the corresponding HRP-conjugated secondary antibodies and finally detected by enhanced chemiluminescence.

\section{SUnSET assay}

Cells were incubated with puromycin $(2.5 \mu \mathrm{g} / \mathrm{ml})$ for $20 \square \min$ and then washed with ice cold PBS and lysed using RIPA lysis buffer. Equal quantity of cell lysates was submitted to western blot using anti-puromycin antibody to detect protein synthesis. Signals were normalized with probing GAPDH and TUBULIN (loading control).

\section{Construction of Stable and inducible Expression Mammalian Cell Lines}

For preparation of lentiviruses, HEK293T cells in 6-well plates were transfected with the lentiviral vector of interest $(1,800 \mathrm{ng})$, the lentiviral packaging plasmids psPAX2 (600 ng) and 
pMD2.G (600 ng) and $12 \mathrm{ul}$ of PEI $(1 \mathrm{mg} / \mathrm{ml})$. About $48 \mathrm{~h}$ after transfection, culture medium containing lentiviruses was collected and centrifugalized at $12,000 \mathrm{rpm}$ for $10 \mathrm{~min}$, and then filtered using 0.22 um filter. HEK293T, N2a and U87 cells were then infected at $\sim 50 \%$ confluency by lentiviruses for $48 \mathrm{~h}$, followed by selection with puromycin or hygromycin for 7 days. Monoclonal cells were obtained by limiting dilution.

\section{Oligonucleotide extension assay}

Total RNA was ligated with oligonucleotide adaptor 1 or 2 respectively using T4 RNA Ligase 1 (M0204S, NEB) following manual. Then RNA was purified by ethanol precipitation and then reverse transcribed using R1 or R2 (R312-02, Vazyme). cDNA was amplified by PCR using $\mathrm{F} 1+\mathrm{R} 1$ or $\mathrm{F} 2+\mathrm{R} 2$. PCR products were firstly purified, then linked into $\mathrm{T}$ vector (CT101-01, Transgen Biotech), and finally sequenced by sanger sequencing.

The sequence of oligonucleotide adapters and primers: adaptor 1 : 5-PO ${ }_{4}$-CTGTAGGCACCATCAATGGACCT-NH ${ }_{2}-3$ (DNA);

adaptor 2 : 5- $\mathrm{NH}_{2}$-CAGAAGGCACCAACAAAGGACC-OH-3 (RNA);

F1: 5-ACCTGGGTATAGGGGCGAAAGAC-3 (DNA)

R1: 5-AGGTCCATTGATGGTGCCTACAG-3 (DNA)

F2: 5-CAGAAGGCACCAACAAAGGACC-3 (DNA)

R2: 5-CCCTTAGAGCCAATCCTTATCCC-3 (DNA)

\section{Reconstitution of the collateral activity of RfXCas13d in vitro}

To detect the collateral activity of RfxCas13d in vitro, we performed in vitro cleavage assay with 100 ng purified RfxCas13d protein, 100 ng synthesized tdTomato RNA, 100 ng crRNA, $2 \mu 1$ RNase inhibitor (New England Biolabs), and $200 \mathrm{ng}$ quenched fluorescent RNA reporters (6 nt polyA/U/G/C), in $100 \mu$ reaction buffer ( $40 \mathrm{mM}$ Tris-HCl, $\left.60 \mathrm{mM} \mathrm{NaCl}, 6 \mathrm{mM} \mathrm{MgCl}_{2}, \mathrm{pH} 7.6\right)^{[5]}$. Reactions were incubated at $37^{\circ} \mathrm{C}$ for $1 \mathrm{~h}$ and measured the fluorescence of RNA reporters with microplate reader. In Fig. S5I, quenched fluorescent RNA reporters were replaced with total RNA extracted from HEK293T cells. Reactions were incubated at $37^{\circ} \mathrm{C}$ for $1 \mathrm{~h}$ and then RNA was purified by ethanol precipitation and quantified by Agilent 2200 Bioanalyzer.

\section{RNA denaturing gel electrophoresis}

Make gel: Weigh $0.5 \mathrm{~g}$ of agarose powder, add it to $36.5 \mathrm{ml}$ of DEPC water, and heat to completely dissolve the agarose. After cooling slightly $\left(60-70^{\circ} \mathrm{C}\right)$, add $5 \mathrm{ml}$ of $10 \mathrm{x}$ MOPS Running Buffer (C516042-0001, Sangon Biotech), $8.5 \mathrm{ml}$ of 37\% formaldehyde. Then pour the gel in the glue tank, insert the comb, and place it horizontally for use after solidification. Add samples: Mix the following reagents in a clean small centrifuge tube: 2 ul 10x MOPS Running buffer, $3.5 \mathrm{ul}$ formaldehyde, $10 \mathrm{ul}$ formamide (deionized), $4.5 \mathrm{ul}$ RNA sample. Mix well, keep it at $60^{\circ} \mathrm{C}$ for $10 \mathrm{~min}$, and cool quickly on ice. Add 3 ul of 10x loading buffer (B548318-0001, Sangon Biotech) and $0.5 \mathrm{ul}$ of ethidium bromide, then mix well and add an appropriate amount to the sample well of the gel. Electrophoresis: Turn on the electrophoresis instrument, and stabilize the electrophoresis at $7.5 \mathrm{~V} / \mathrm{cm}$.

\section{Total RNA integrity analysis}

Total RNA was extracted from cells and then quantified by Agilent 2200 Bioanalyzer.

\section{RNA-seq analysis}

The sequencing data generated by illunima Noveseq PE150 in fastq file format was filtered by FastQC(https://www.bioinformatics.babraham.ac.uk/projects/fastqc/) and Trim-Galore(https://www.bioinformatics.babraham.ac.uk/projects/trim_galore/) softwares for quality control. Then the mouse genome version $\mathrm{mm} 10$ and the human genome version $\mathrm{hg} 38$ were 
512 used as reference genome to align the clean data with Subread software 513 (http://subread.sourceforge.net/). The gene count matrix was calculated by the featureCounts 514 (http://subread.sourceforge.net/) program. Then the gene count data was normalized using the 515 FPKM formula. The differentially expressed genes were analysed by $\mathrm{R}$ package DESeq2 516 (https://bioconductor.org/packages/release/bioc/vignettes/DESeq2/inst/doc/DESeq2.html).

517 Transcription factor enrichment analysis was conducted by ChEA3

518 (https://maayanlab.cloud/chea3/\#top). The raw data and processed data were uploaded to the GEO 519 Datasets (GSE193668).

\section{Statistical Analysis}

521 The descriptive statistical analysis was performed with Prism version 8 (GraphPad Software). All 522 data are presented as mean \pm SEM. A two-tailed Student's t test assuming equal variants was used 523 to compare two groups. In all figures, the statistical significance between the indicated samples 524 and control is designated as $* \mathrm{P}<0.05$, $* * \mathrm{P}<0.01$, *** $\mathrm{P}<0.001$, or NS $(\mathrm{P}>0.05)$ 

Nat Rev Microbiol 2020; 18(2):67-83. 2015; 60(3):385-397. targeting with CRISPR-Cas13. Nature 2017; 550(7675):280-284. the rise of CRISPR-resistant bacteriophage. Nature 2019; 570(7760):241-245. acid detection with CRISPR-Cas13a/C2c2. Science 2017; 356(6336):438-442. editing with CRISPR-Cas13. Science 2017; 358(6366): 1019-1027.

540 7. Shmakov S, Smargon A, Scott D, Cox D, Pyzocha N, Yan W, et al. Diversity and evolution

541 of class 2 CRISPR-Cas systems. Nat Rev Microbio/2017; 15(3):169-182.

542 8. Konermann S, Lotfy P, Brideau NJ, Oki J, Shokhirev MN, Hsu PD. Transcriptome

543 Engineering with RNA-Targeting Type VI-D CRISPR Effectors. Cell 2018; 173(3):665-676 544 e614.

545 9. Yan WX, Chong S, Zhang H, Makarova KS, Koonin EV, Cheng DR, et al. Cas13d Is a 
547 WYL-Domain-Containing Accessory Protein. Mol Cel/2018; 70(2):327-339 e325.

548 10. Xu C, Zhou Y, Xiao Q, He B, Geng G, Wang Z, et al. Programmable RNA editing with

549 compact CRISPR-Cas13 systems from uncultivated microbes. Nat Methods 2021;

550 18(5):499-506.

551 11. Zhou H, Su J, Hu X, Zhou C, Li H, Chen Z, et al. Glia-to-Neuron Conversion by

552 CRISPR-CasRx Alleviates Symptoms of Neurological Disease in Mice. Cell 2020;

553 181(3):590-603 e516.

554 12. He B, Peng W, Huang J, Zhang H, Zhou Y, Yang X, et al. Modulation of metabolic

555 functions through Cas13d-mediated gene knockdown in liver. Protein Cell 2020;

556 11(7):518-524.

557 13. Zhou C, Hu X, Tang C, Liu W, Wang S, Zhou Y, et al. CasRx-mediated RNA targeting

558 prevents choroidal neovascularization in a mouse model of age-related macular degeneration.

$559 \quad$ Nat/ Sci Rev 2020; 7(5):835-837.

560 14. Wang Q, Liu X, Zhou J, Yang C, Wang G, Tan Y, et al. The CRISPR-Cas13a Gene-Editing

561 System Induces Collateral Cleavage of RNA in Glioma Cells. Adv Sci (Weinh) 2019;

$562 \quad 6(20): 1901299$.

563 15. Zhang Z, Wang Q, Liu Q, Zheng Y, Zheng C, Yi K, et al. Dual-Locking Nanoparticles

564 Disrupt the PD-1/PD-L1 Pathway for Efficient Cancer Immunotherapy. Adv Mater 2019;

565 31(51):e1905751.

566 16. Wang L, Zhou J, Wang Q, Wang Y, Kang C. Rapid design and development of

567 CRISPR-Cas13a targeting SARS-CoV-2 spike protein. Theranostics 2021; 11(2):649-664. 
569 RNA targeting with the single-protein CRISPR effector Cas7-11. Nature 2021;

$570 \quad 597(7878): 720-725$.

571 18. Funato $H$, Miyoshi $C$, Fujiyama $T$, Kanda $T$, Sato $M$, Wang Z, et al. Forward-genetics

572 analysis of sleep in randomly mutagenized mice. Nature 2016; 539(7629):378-383.

573 19. Wang G, Li Q, Xu J, Zhao S, Zhou R, Chen Z, et al. 2021.

574 20. Chan KY, Jang MJ, Yoo BB, Greenbaum A, Ravi N, Wu WL, et al. Engineered AAVs for

575 efficient noninvasive gene delivery to the central and peripheral nervous systems. Nat

576 Neurosci2017; 20(8):1172-1179.

577 21. Sasagawa S, Takemori H, Uebi T, Ikegami D, Hiramatsu K, Ikegawa S, et al. SIK3 is 578 essential for chondrocyte hypertrophy during skeletal development in mice. Development $579 \quad 2012 ; 139(6): 1153-1163$.

580 22. Hayasaka N, Hirano A, Miyoshi Y, Tokuda IT, Yoshitane H, Matsuda J, et al. Salt-inducible 581 kinase 3 regulates the mammalian circadian clock by destabilizing PER2 protein. Elife 2017; 6.

582 23. Wang HY, Hsieh PF, Huang DF, Chin PS, Chou CH, Tung CC, et al. RBFOX3/NeuN is 583 Required for Hippocampal Circuit Balance and Function. Sci Rep 2015; 5:17383.

584 24. Teng J, Takei Y, Harada A, Nakata T, Chen J, Hirokawa N. Synergistic effects of MAP2 585 and MAP1B knockout in neuronal migration, dendritic outgrowth, and microtubule organization. $586 J$ J Cell Biol 2001; 155(1):65-76.

587 25. Gumucio A, Lannfelt L, Nilsson LN. Lack of exon 10 in the murine tau gene results in mild 588 sensorimotor defects with aging. BMC Neurosci2013; 14:148. 
589

26. Vicente MM, Chaves-Ferreira M, Jorge JMP, Proenca JT, Barreto VM. The Off-Targets of

590 Clustered Regularly Interspaced Short Palindromic Repeats Gene Editing. Front Cell Dev Biol

$591 \quad 2021 ; 9: 718466$.

592

593

594

595

596

597

598

599

600

601

602

603

604

605

606

607

608

609

27. Madisen L, Zwingman TA, Sunkin SM, Oh SW, Zariwala HA, Gu H, et al. A robust and

high-throughput Cre reporting and characterization system for the whole mouse brain. Nat Neurosci 2010; 13(1):133-140.

28. Vialetto E, Yu Y, Collins SP, Wandera KG, Barquist L, Beisel CL. 2021.

29. East-Seletsky A, O'Connell MR, Knight SC, Burstein D, Cate JH, Tjian R, et al. Two distinct

RNase activities of CRISPR-C2c2 enable guide-RNA processing and RNA detection. Nature 2016; 538(7624):270-273.

30. Brogan DJ, Chaverra-Rodriguez D, Lin CP, Smidler AL, Yang T, Alcantara LM, et al. A Sensitive, Rapid, and Portable CasRx-based Diagnostic Assay for SARS-CoV-2. medRxiV 2020.

31. Bahrami S, Drablos F. Gene regulation in the immediate-early response process. Adv Biol Regu/2016; 62:37-49.

32. Vind AC, Genzor AV, Bekker-Jensen S. Ribosomal stress-surveillance: three pathways is a magic number. Nucleic Acids Res 2020; 48(19):10648-10661.

33. Yang J, Shibu MA, Kong L, Luo J, BadrealamKhan F, Huang Y, et al. Design, Synthesis, and Structure-Activity Relationships of 1,2,3-Triazole Benzenesulfonamides as New Selective Leucine-Zipper and Sterile-alpha Motif Kinase (ZAK) Inhibitors. J Med Chem 2020; 63(5):2114-2130. 
bioRxiv preprint doi: https://doi.org/10.1101/2022.01.17.476700; this version posted January 18, 2022. The copyright holder for this preprint (which was not certified by peer review) is the author/funder. All rights reserved. No reuse allowed without permission.

\section{0}




\section{Figure 1}

a

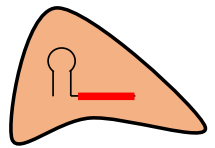

RfxCas13d-crRNA Sik3-L and Sik3-S

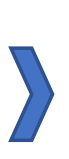

b

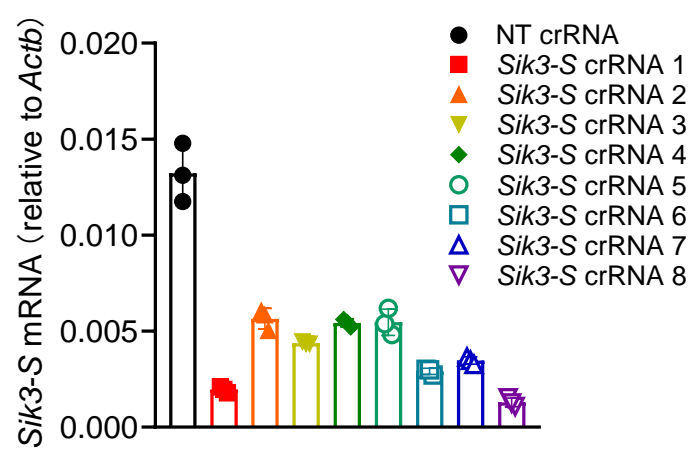

d

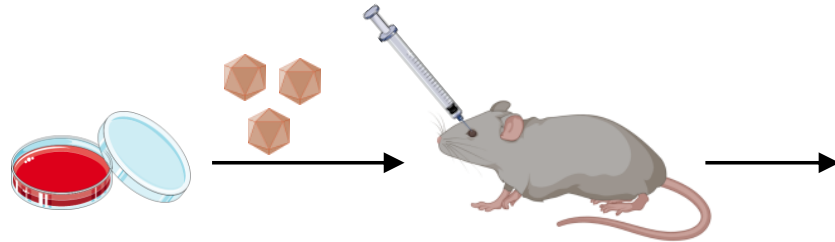

AAV-PHP.eB packaging

Retro-orbital injection

C

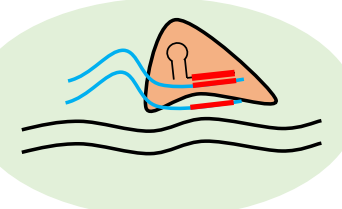

Matching

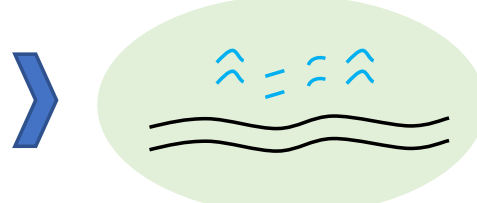

Cleavage

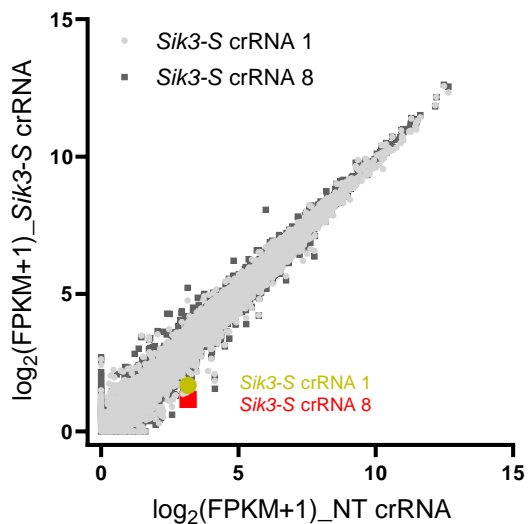

Sik3-S knockdown in neurons Sleep behavior analysis

e

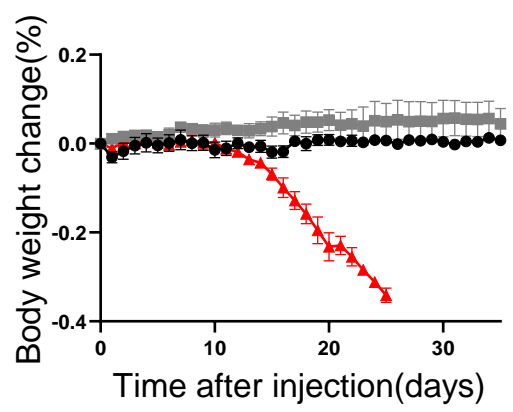

f

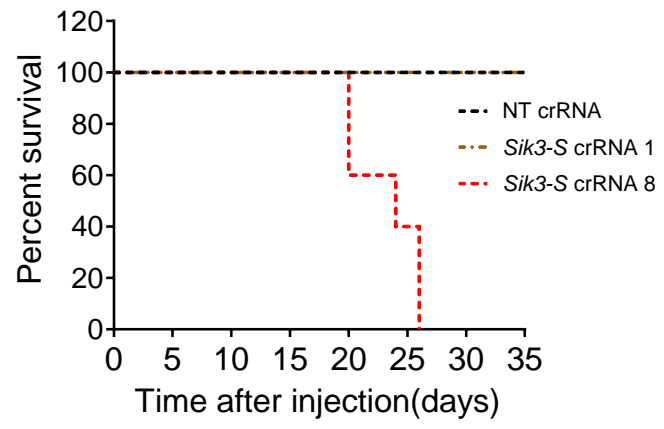

g

$\rightarrow$ NT crRNA

-- Sik3-S crRNA 1

- Sik3-S crRNA 8

\section{Sik3-S}

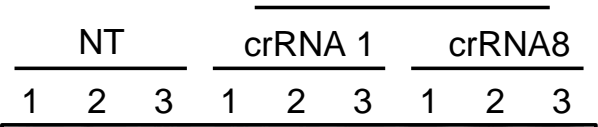

SIK3-S

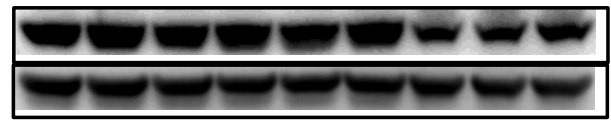




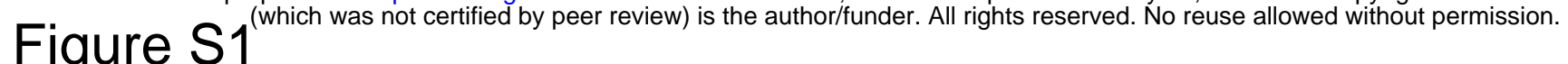

a

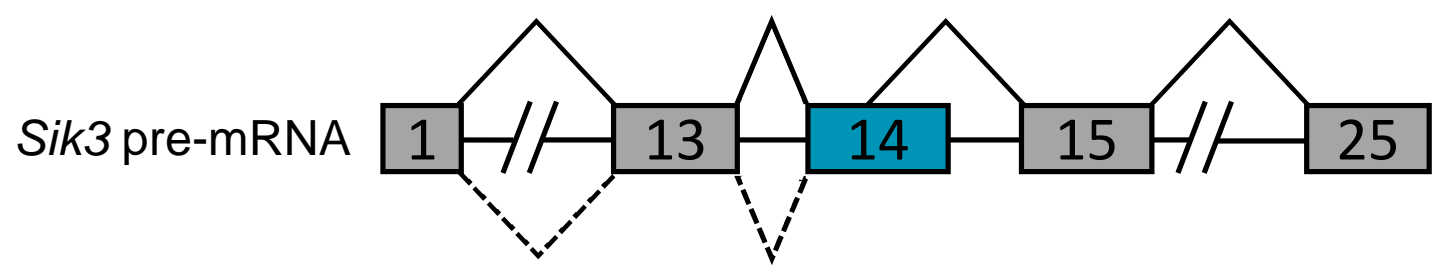

Sik3-L

Sik3-S

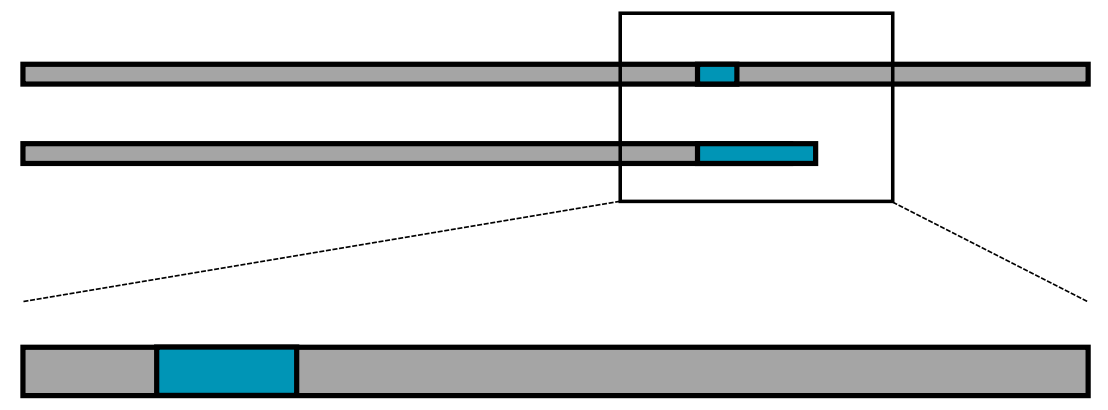

b

Cas130

cr

C

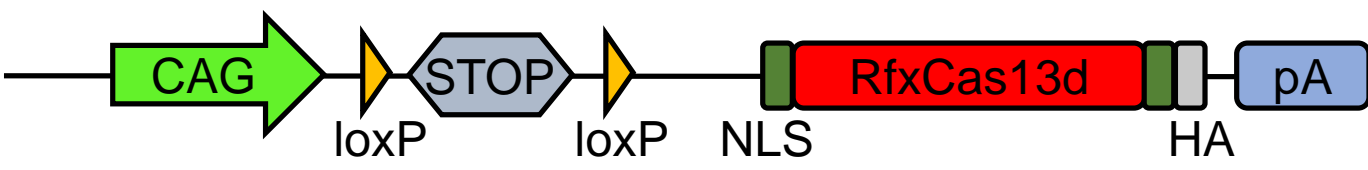

d

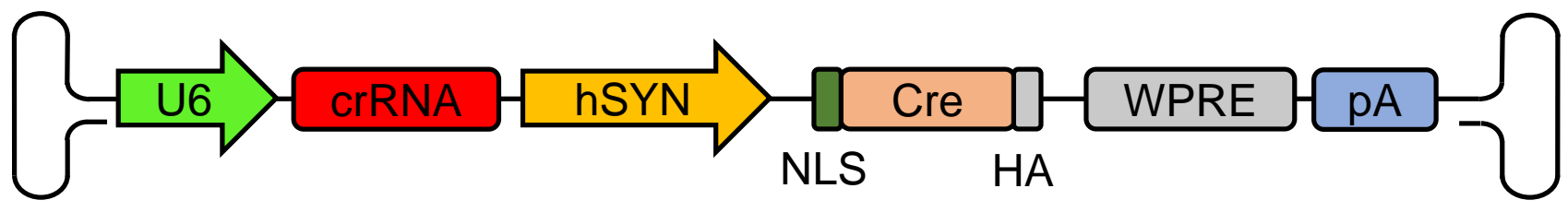


Figure 2

a

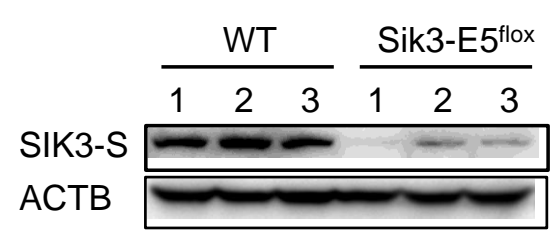

d

g
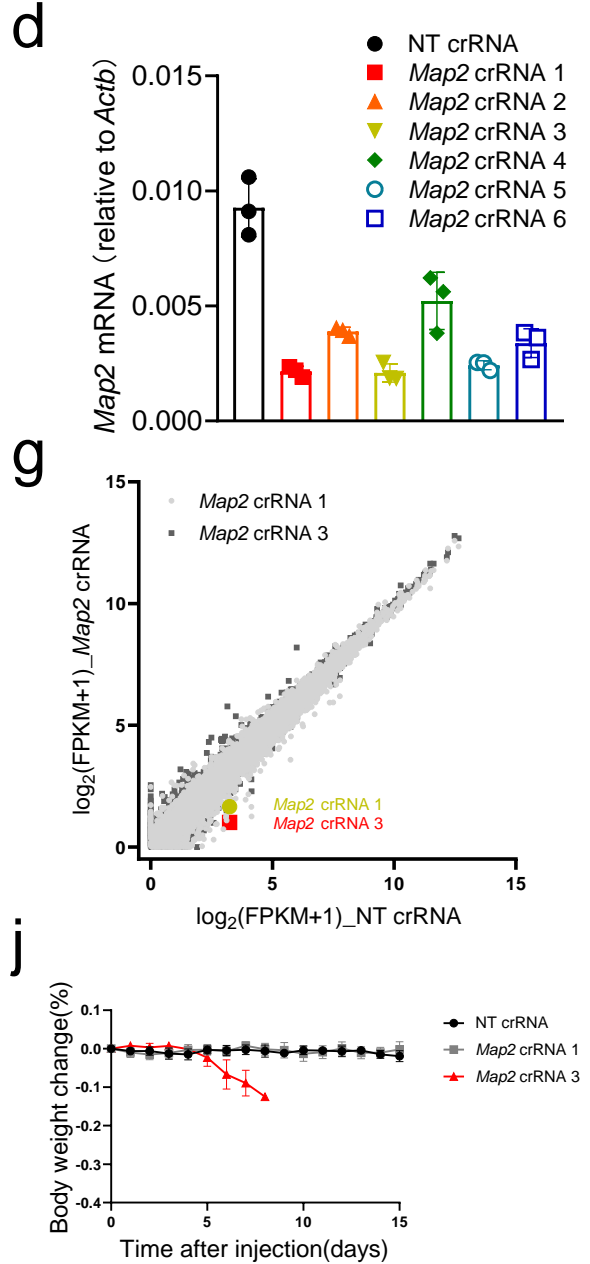

m

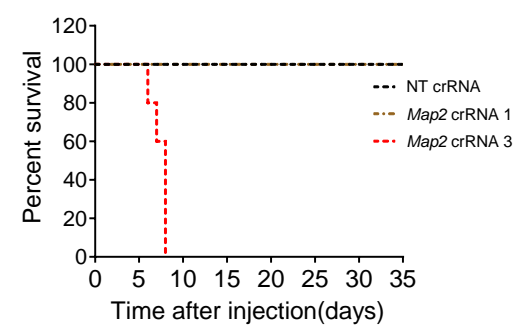

$p$

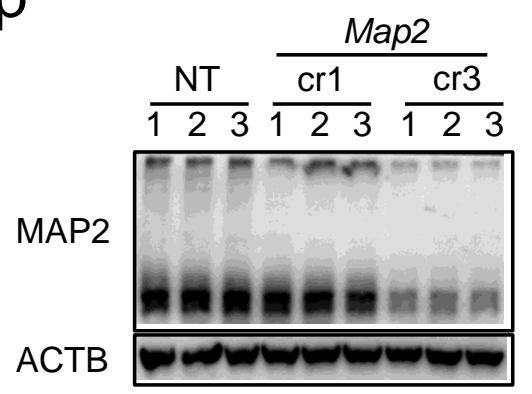

b

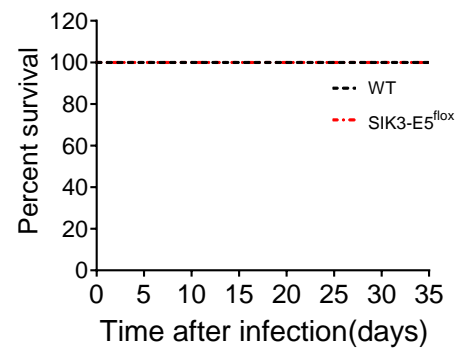

e

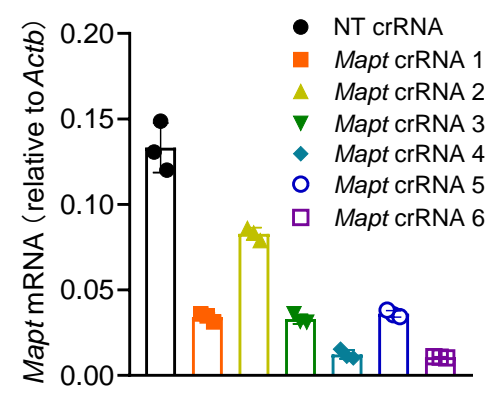

k

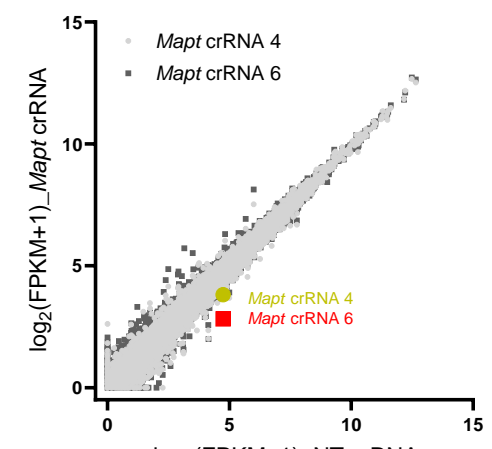

,

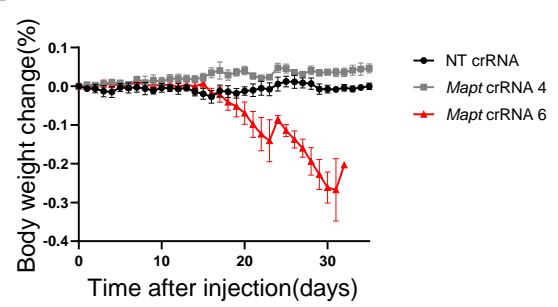

$\mathrm{n}$

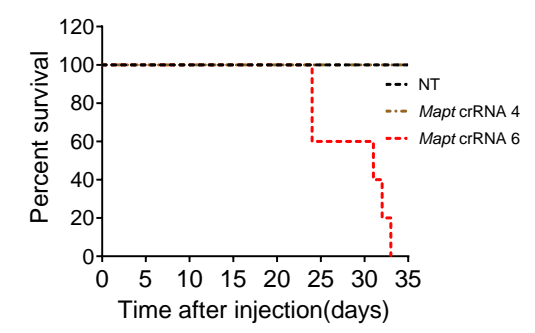

$q$

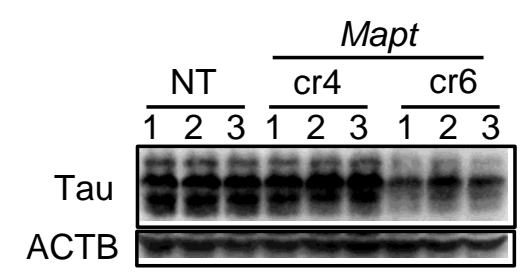

C

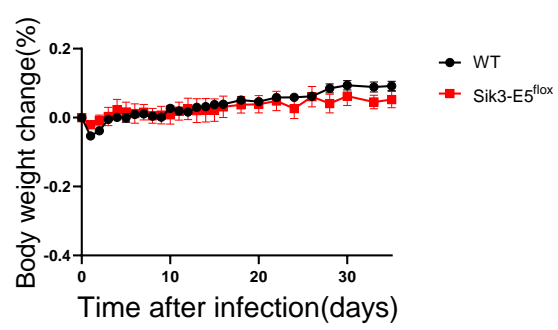

f
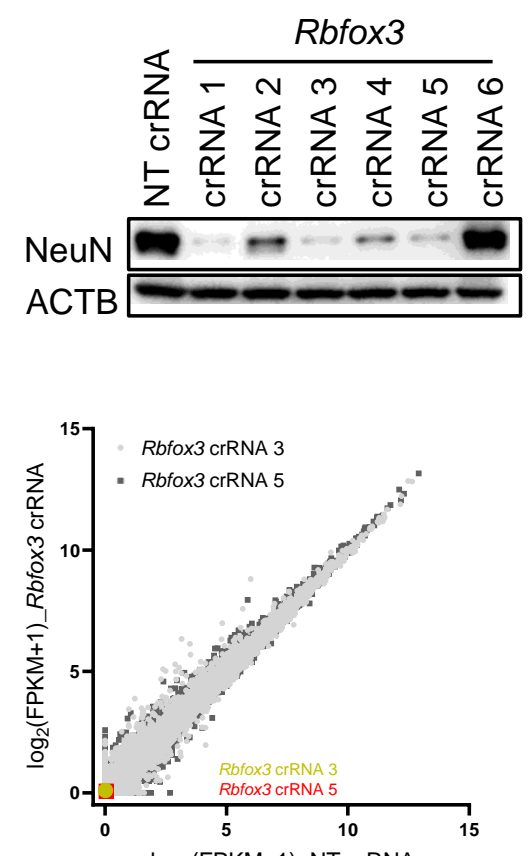

$\log _{2}($ FPKM+1)_NT crRNA

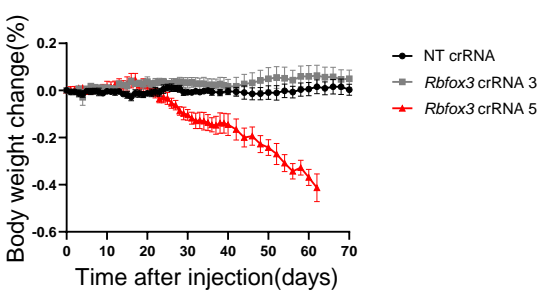

0

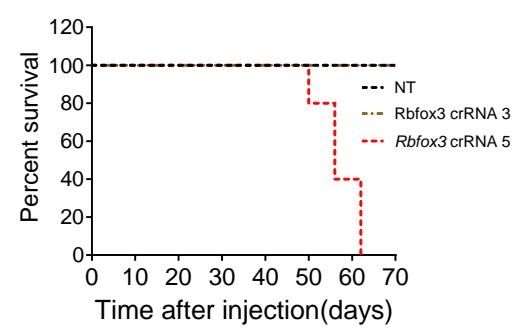

$r$ 
Figure S2

a

Sik3-E5 flox locus

$1-1 / 2$

AAV-PHP.eB-hSYN-Cre

Sik3-E5 locus

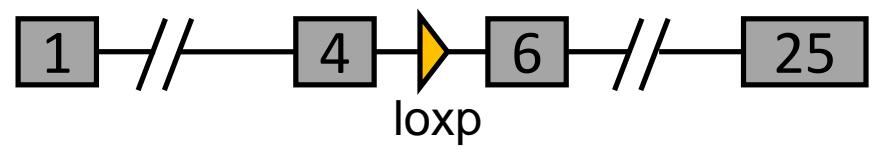


Figure 3

a

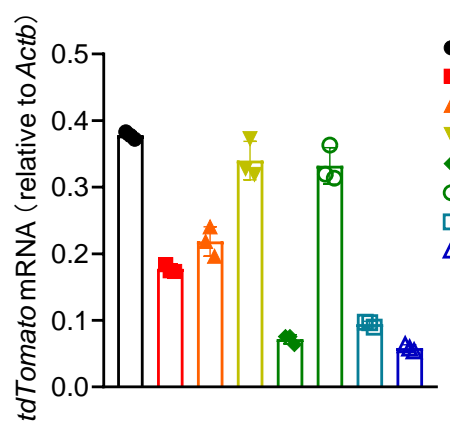

C

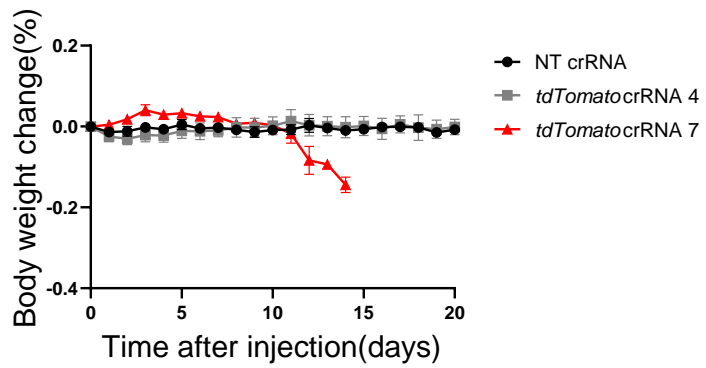

e

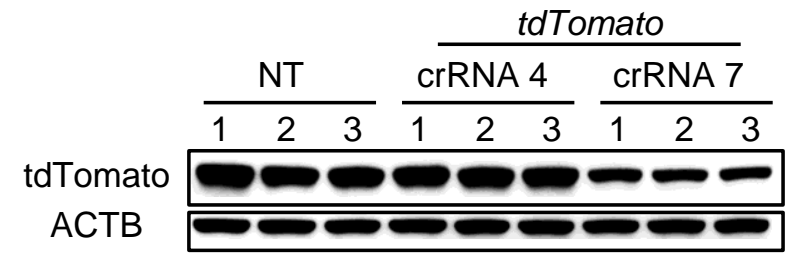

f
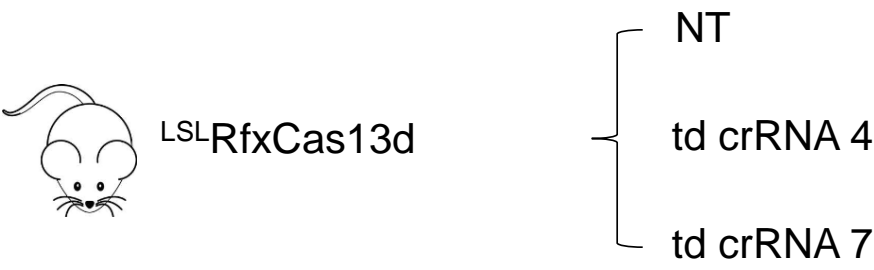

NT

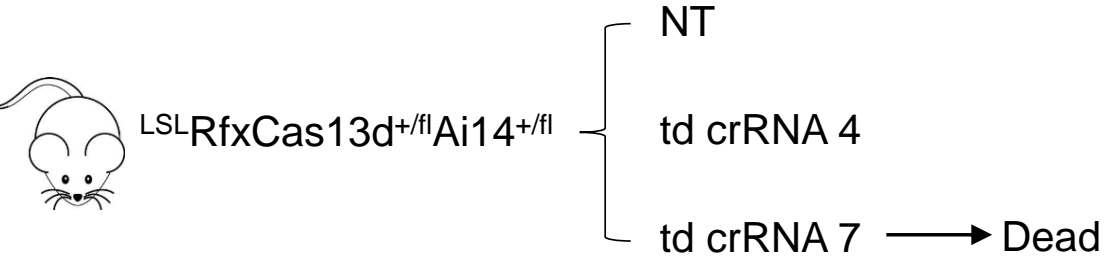

g

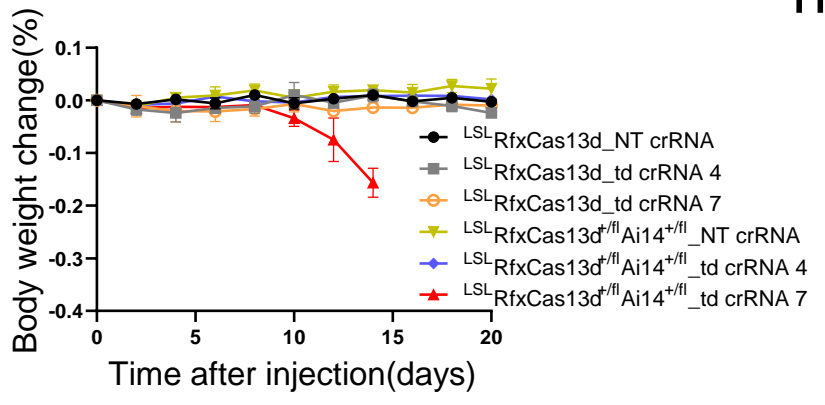

b
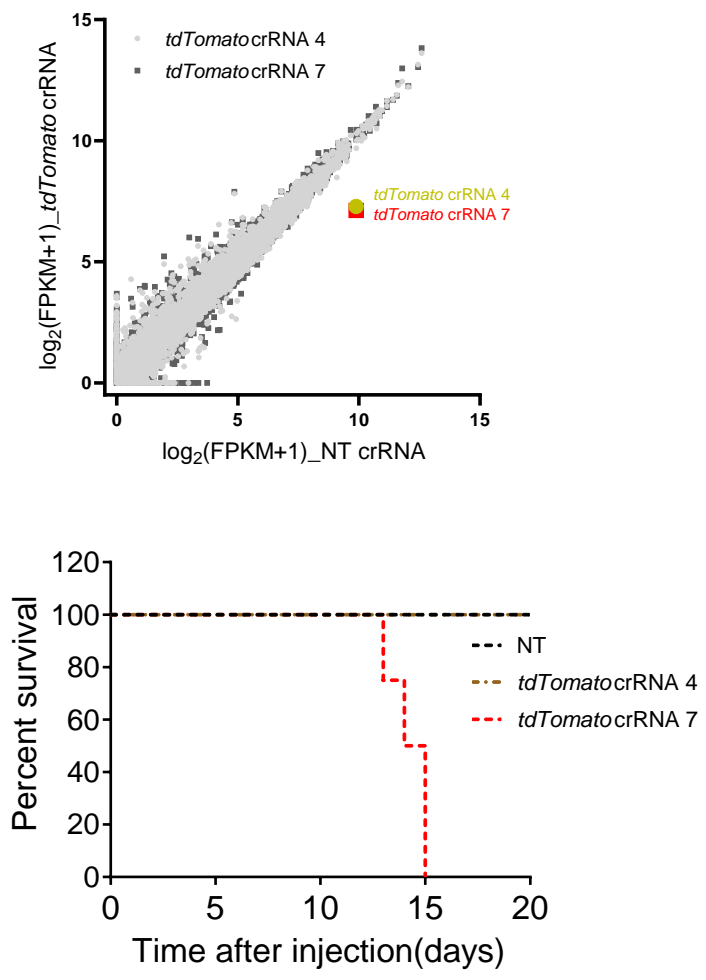
Figure S3

a

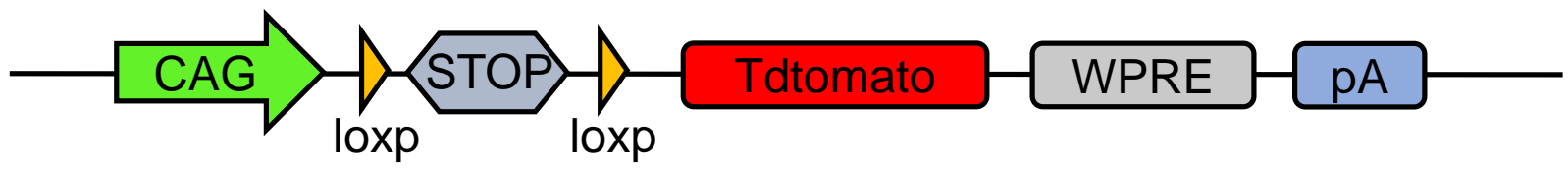

b

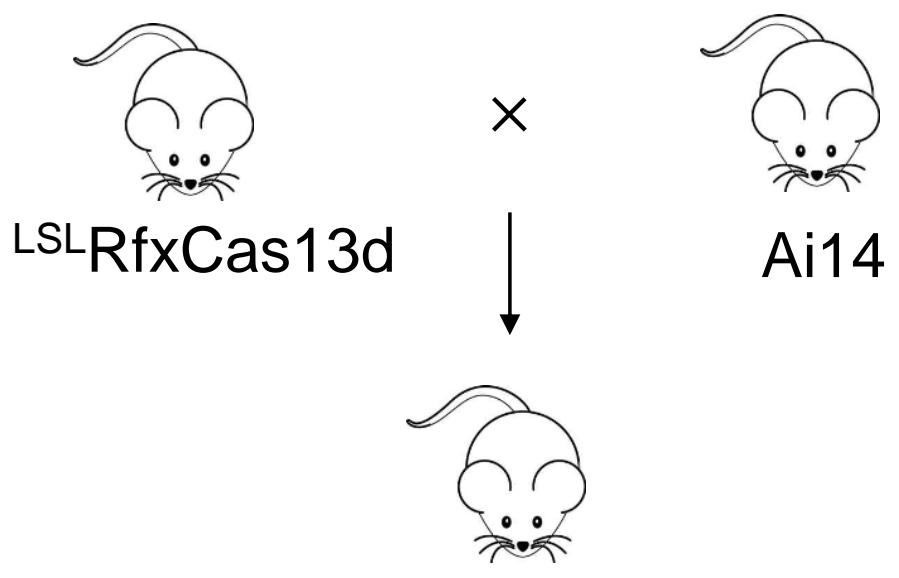

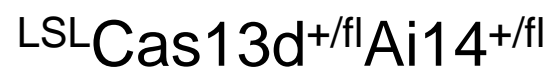


Figure 4

a

$\begin{array}{cccccccccc}\text { RfxCas13d } & + & + & + & + & + & + & \mathrm{d} & \mathrm{d} & \mathrm{d} \\ \text { tdTomato } & - & - & - & + & + & + & + & + & + \\ \text { crRNA } & \mathrm{N} & 4 & 7 & \mathrm{~N} & 4 & 7 & \mathrm{~N} & 4 & 7\end{array}$

RfXCas13d-HA
3F-tdTomato
ACTB

C

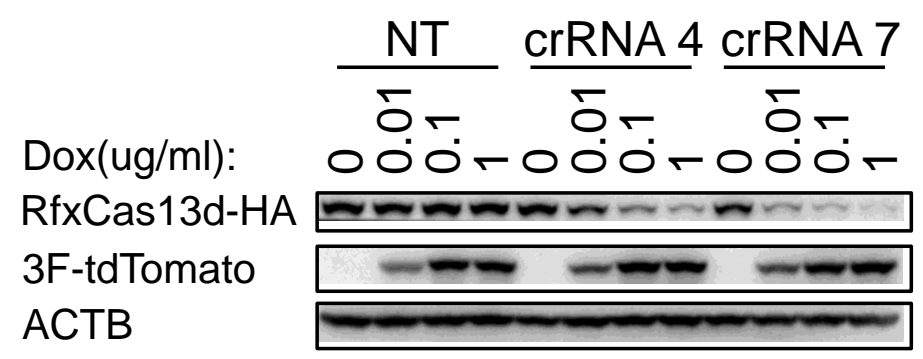

b

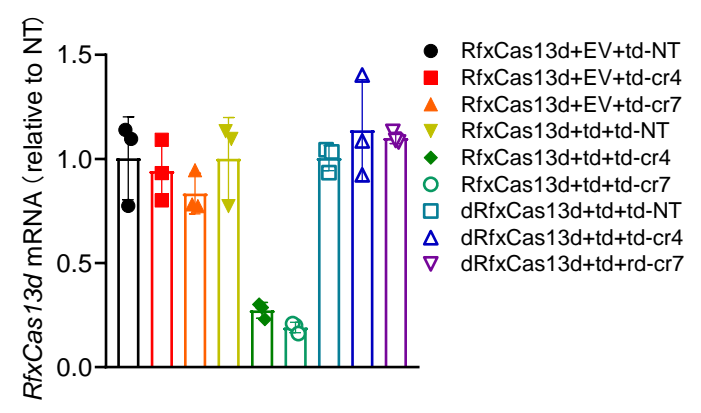

d

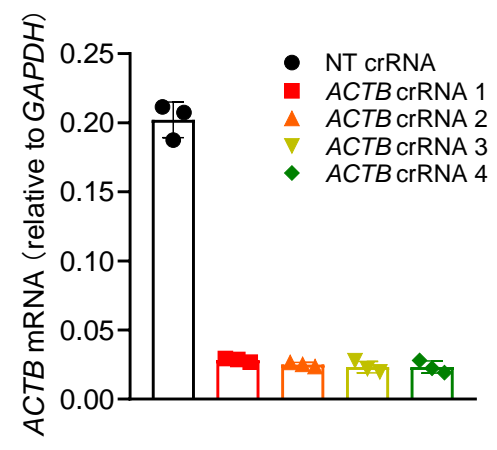

e
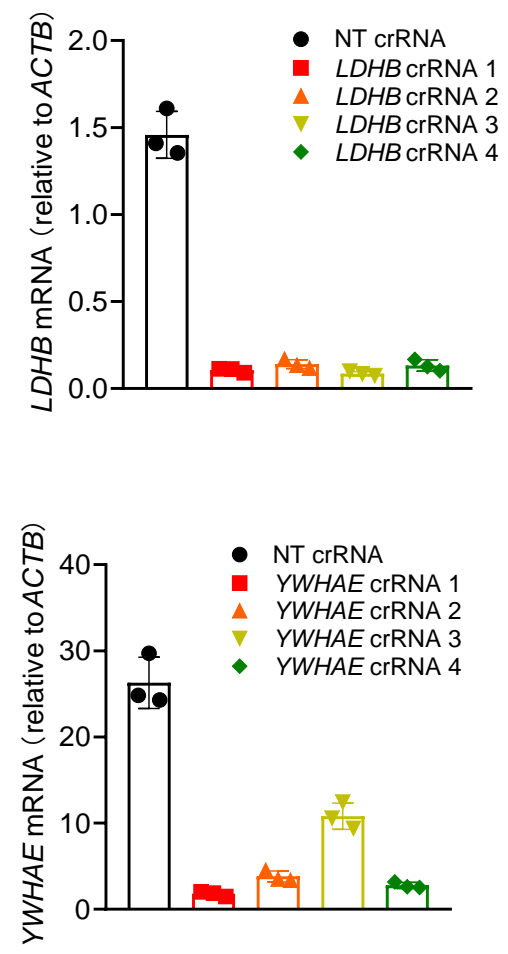

g

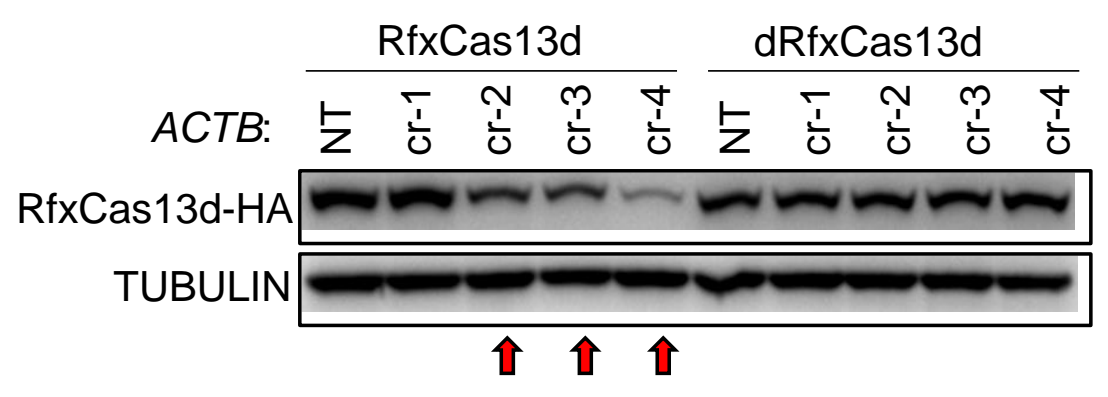

h

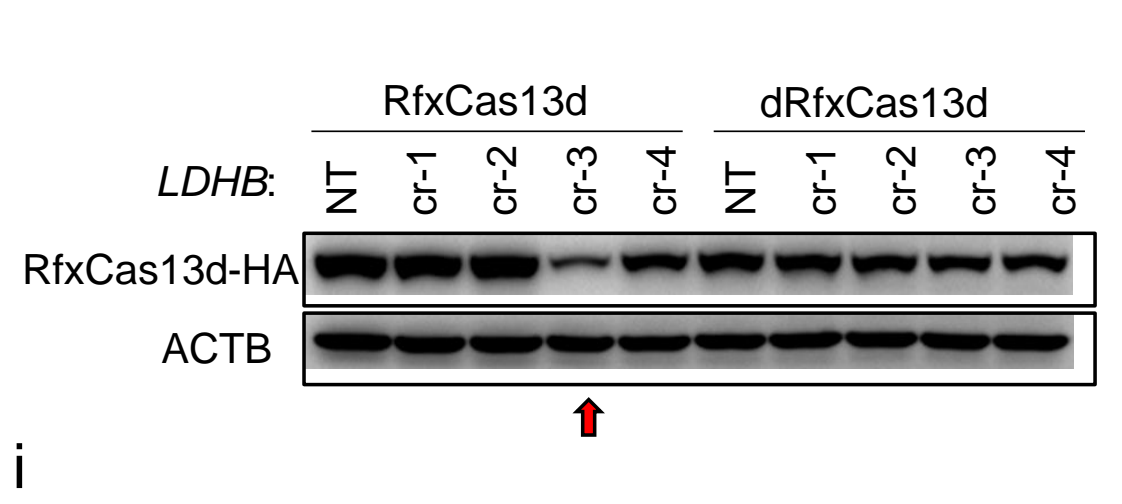

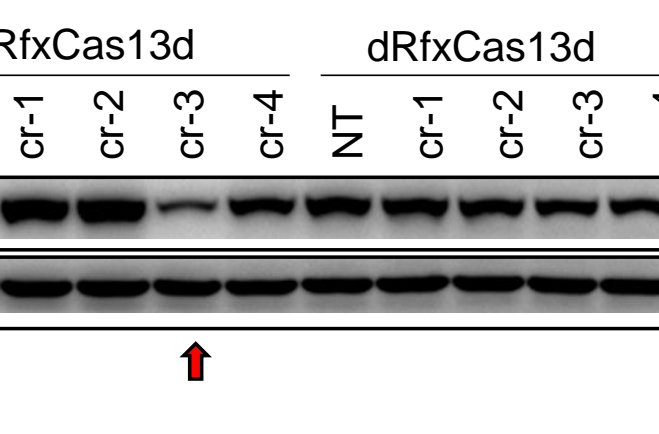

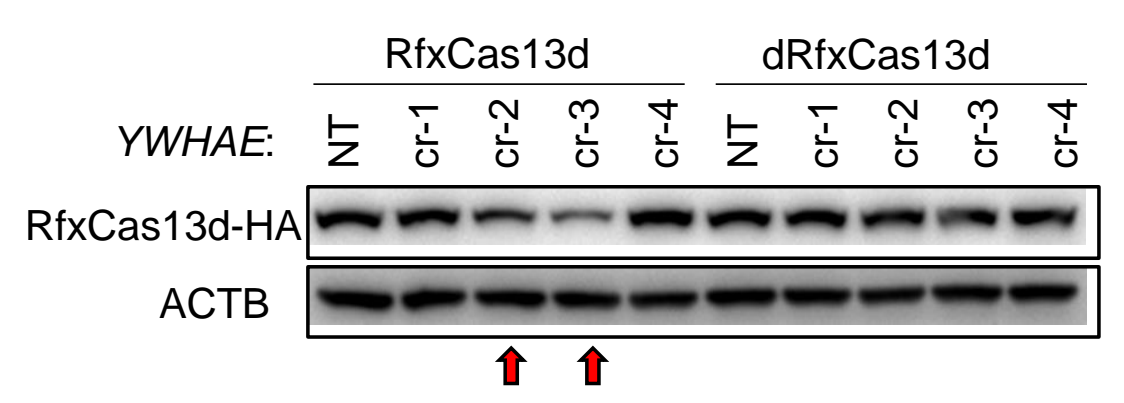


Figure S4

a

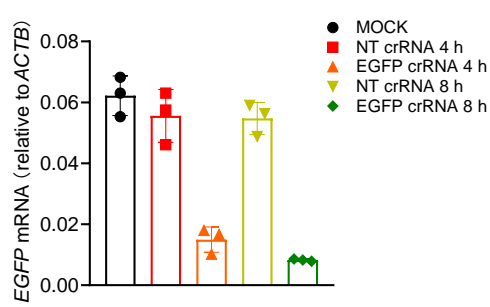

C b

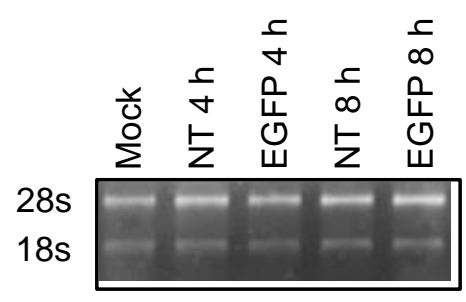

e d

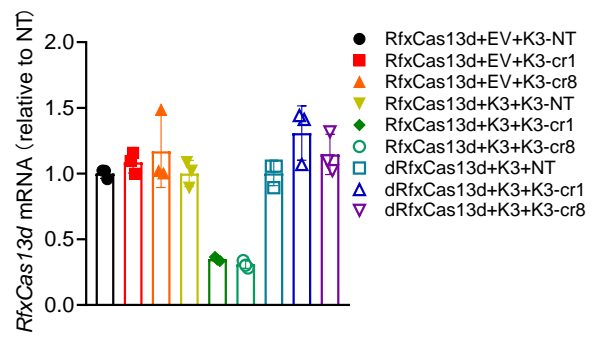

f $\begin{array}{rlllllllll}\text { RfxCas13d: } & + & + & + & + & + & + & \mathrm{d} & \mathrm{d} & \mathrm{d} \\ \text { SIK3-S: } & - & - & - & + & + & + & + & + & + \\ \text { crRNA: } & \mathrm{N} & 1 & 8 & \mathrm{~N} & 1 & 8 & \mathrm{~N} & 1 & 8\end{array}$

$\mathrm{RfxCas} 13 \mathrm{~d}-\mathrm{HA}=\square-\square$

SIK3-S-HA

ACTB

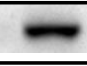

त्व

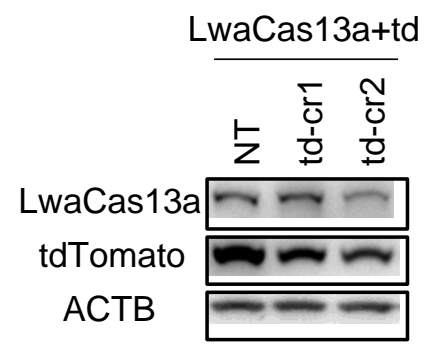

LwaCas $13 a+t d$

PspCas13b+td

乞 $\begin{array}{cc}\overline{1} & \frac{1}{0} \\ \frac{1}{2} & \frac{1}{0}\end{array}$

PspCas13b

tdTomato

ACTB g

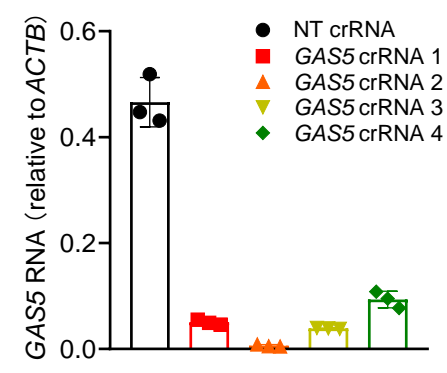

h
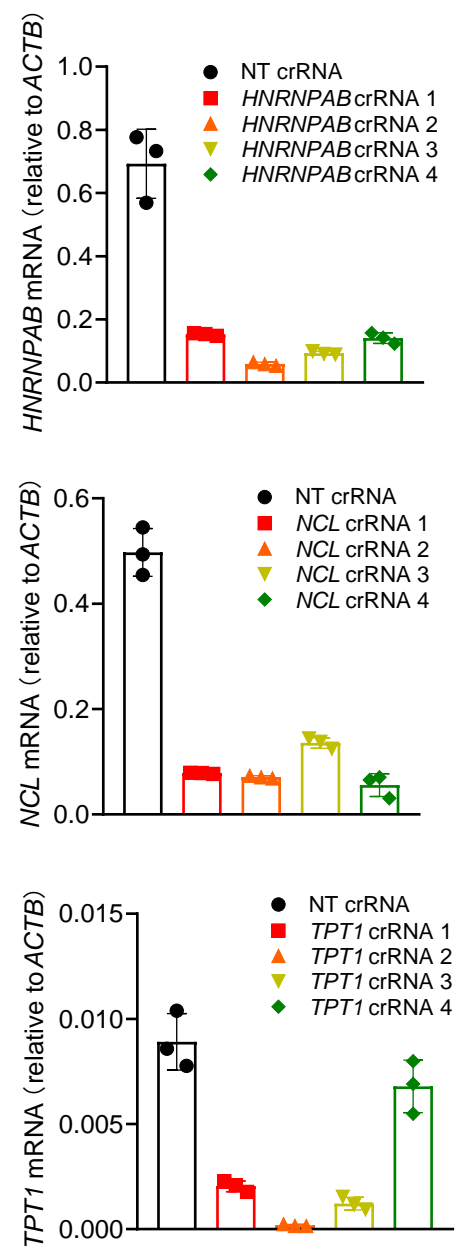

n

k

$\mathrm{m}$
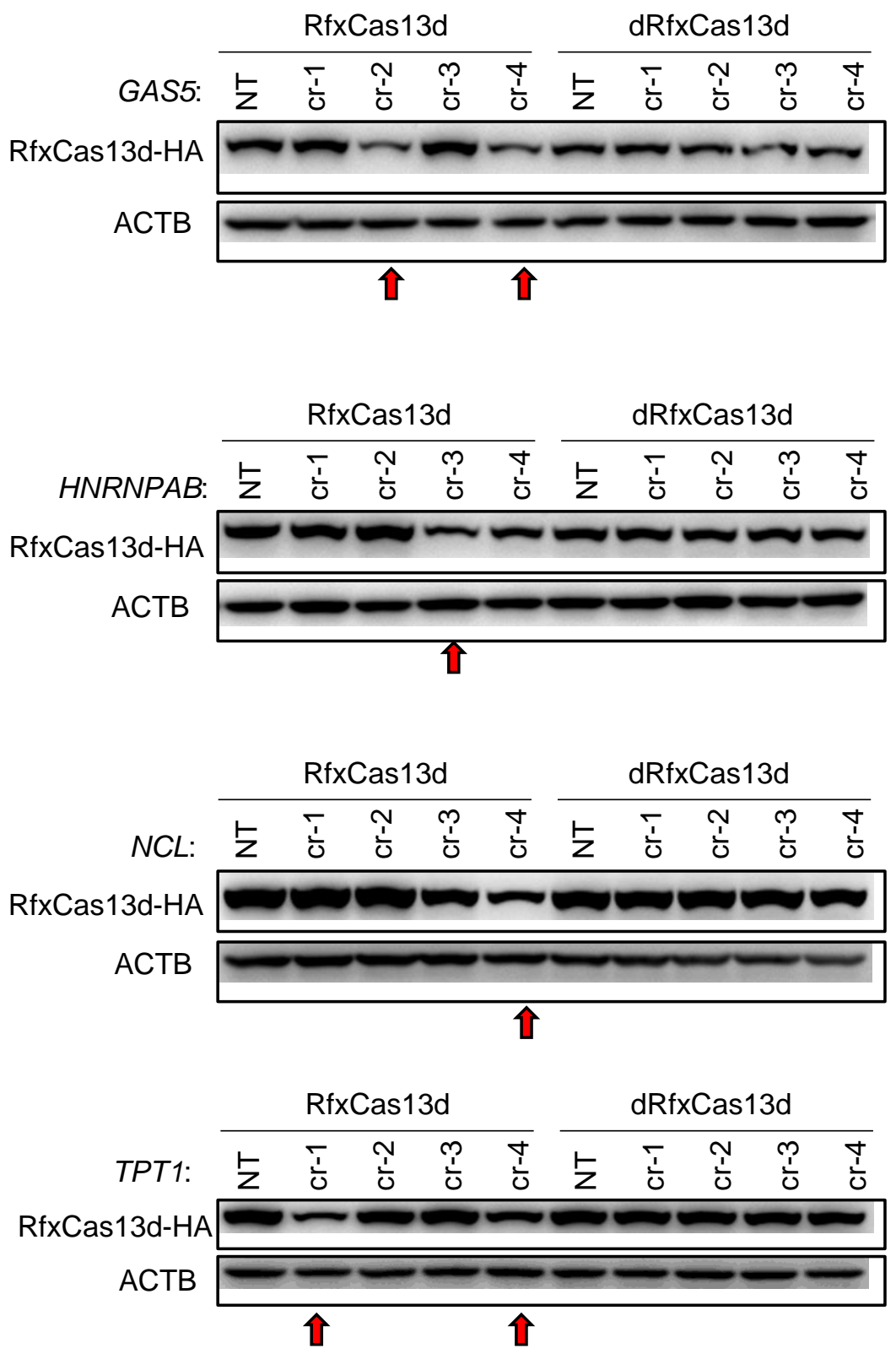
Figure 5

a

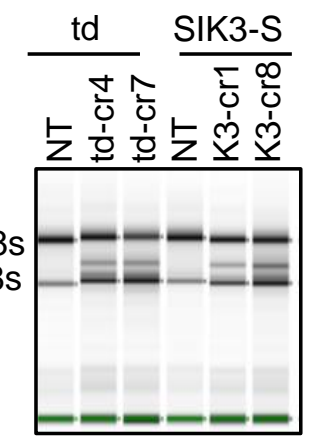

b

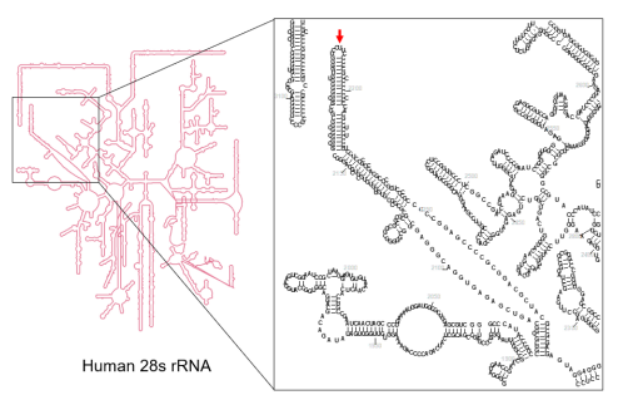

d

g
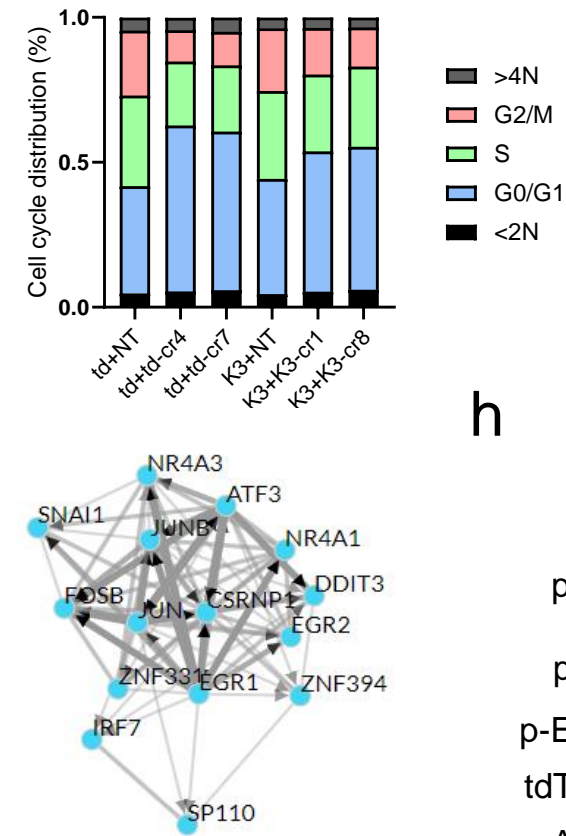

i
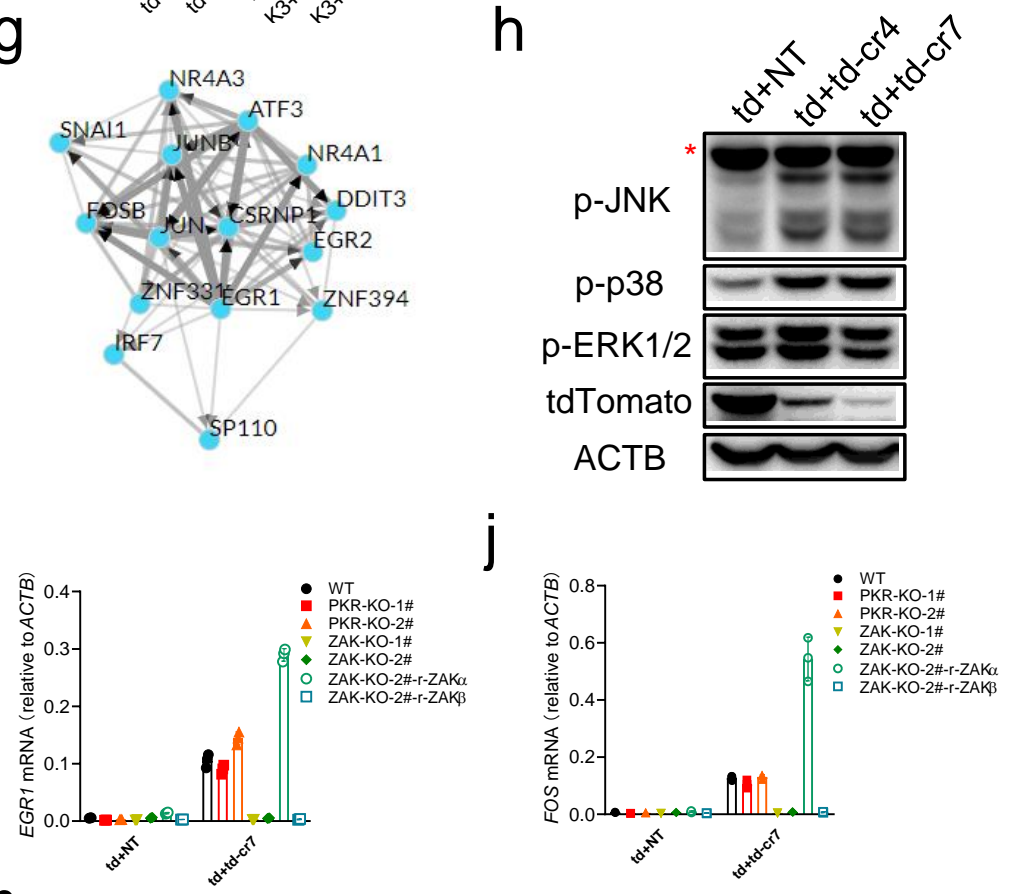

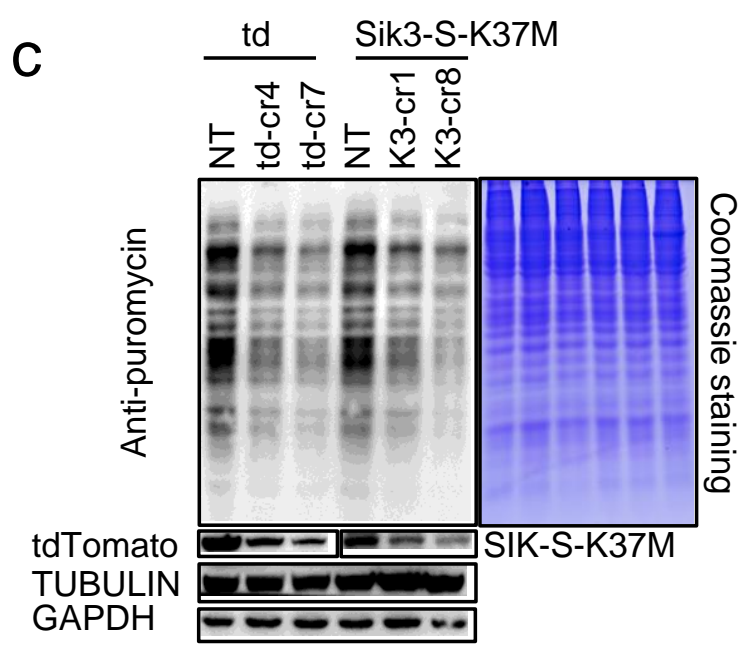

e NT vs td-cr4 NT vs td-cr7

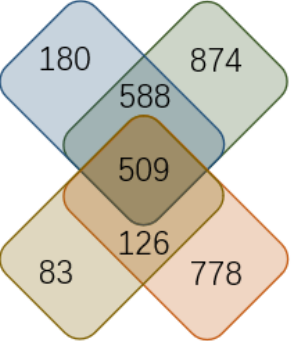

NT vs K3-cr1 NT vs K3-cr8

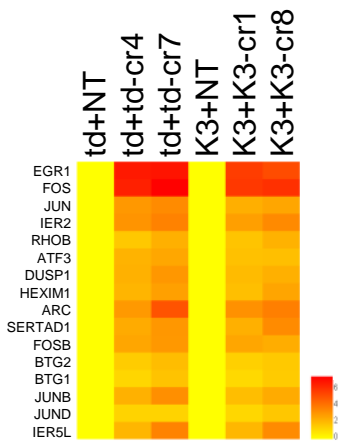

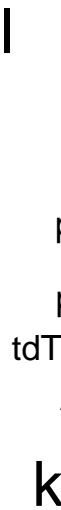

k

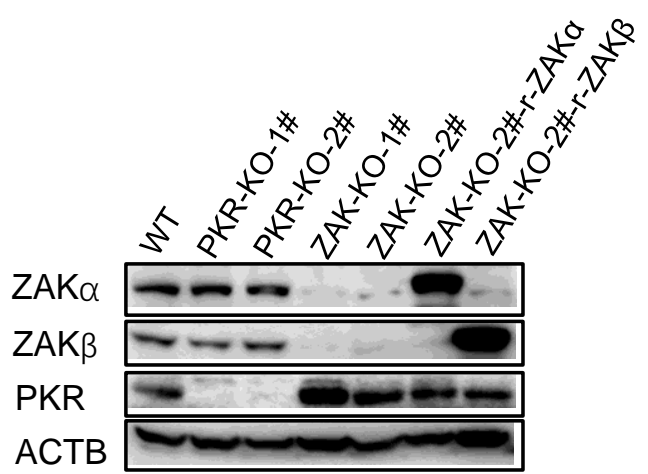

p-JNK

p-p38

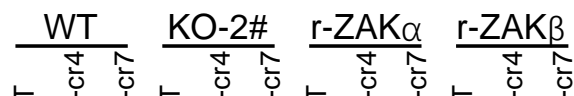

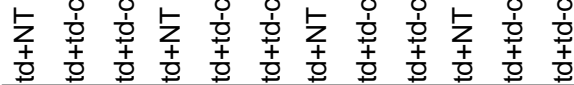

Tomato

ACTB
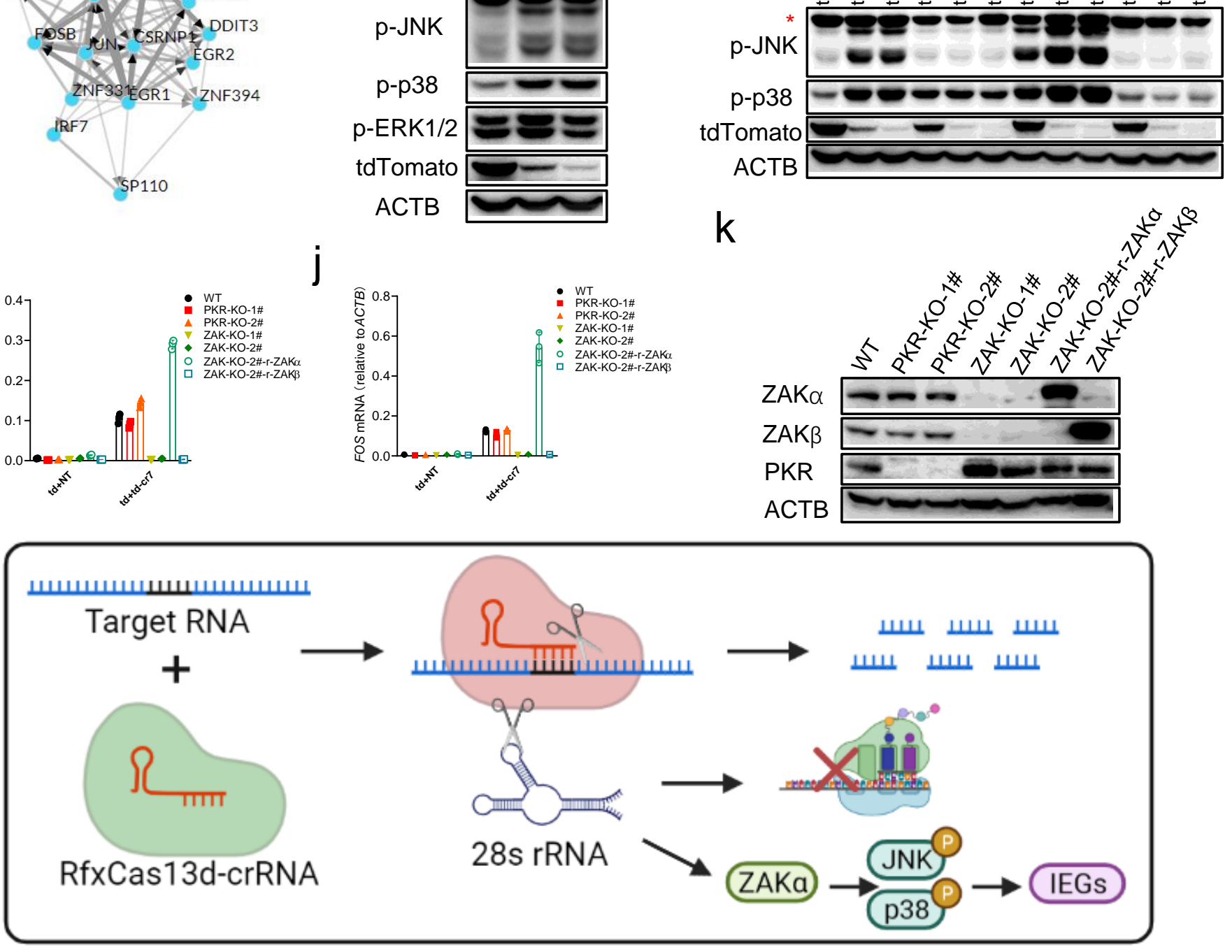
Figure S5

a

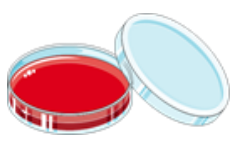

HEK293T-RfxCas13d

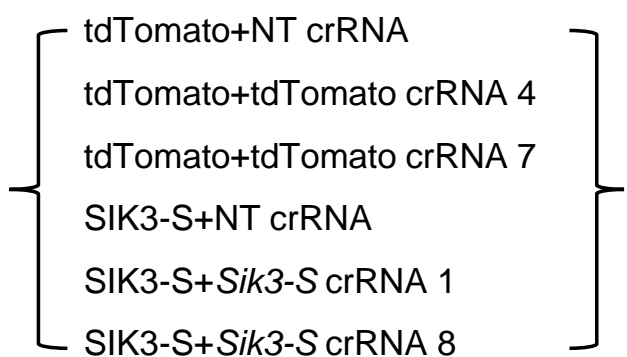

b

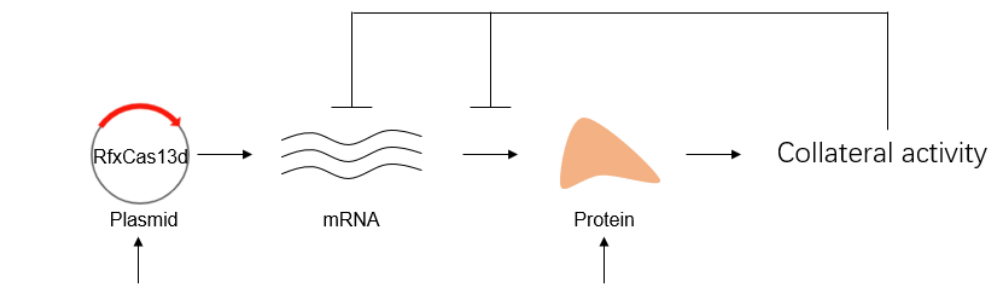

Transient transfection

Stable transfection

d

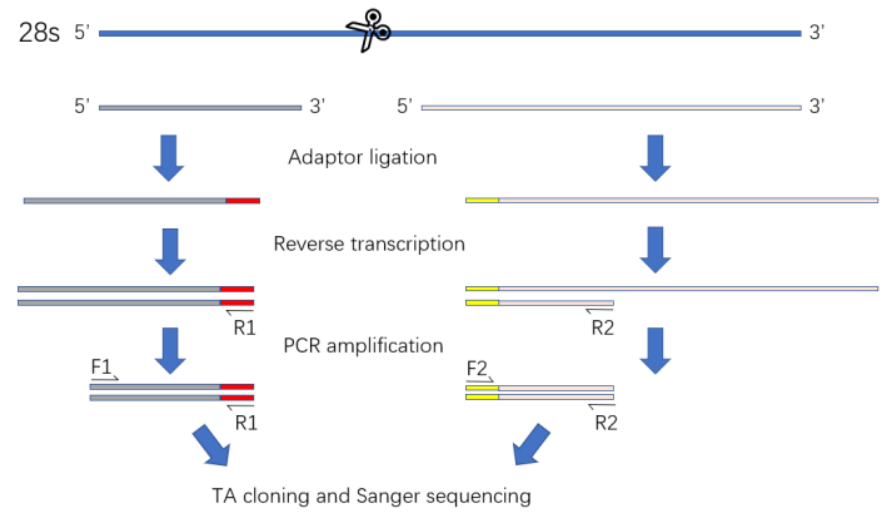

Total RNA integrity analysis

Cell cycle distribution analysis

RNA-seq

C

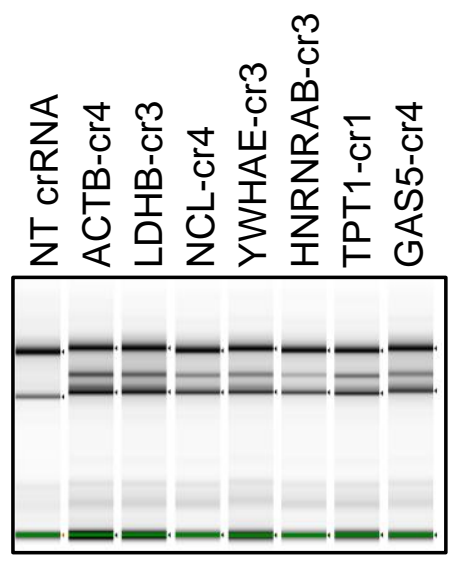

e

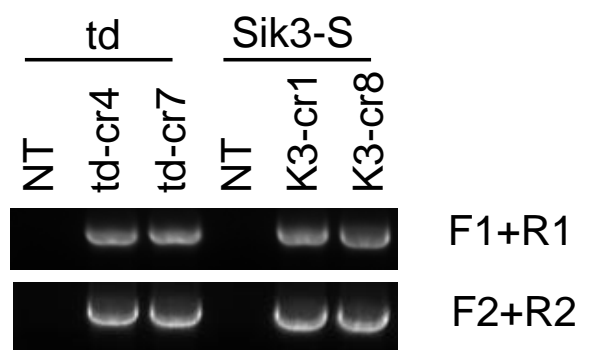

f

\section{$F 1+R 1$ sequencing}

Ref CCCATACCCGGCCGTCGCCGGCAGTCGAGAGTGGACGGGAGCGGCGGGGGCGGCGCGCGCGCGCGCGCGTGTGGTGTGCGTCGGAGGGCGGCGGCGGCGGCGGCGGCGGGGGTGTGGGGTCCTTCCCCCCC..... CCCATACCCGGCCGTCGCCGGCAGTCGAGAGTGGACGGGAGCGGCGGGGGCGGCGCGCGCGCGCGCGCGTGTGGTGTGCGTCGGAGGGCGGCGGCGGCGGCGGCGGCGGGGGTGTGGGGTCCAAAAAATATTAAAA CCCATACCCGGCCGTCGCCGGCAGTCGAGAGTGGACGGGAGCGGCGGGGGCGGCGCGCGCGCGCGCGCGTGTGGTGTGCGTCGGAGGGCGGCGGCGGCGGCGGCGGCGGCGGGGGTGTGTGGGGTCCT CCCATACCCGGCCGTCGCCGGCAGTCGAGAGTGGACGGGAGCGGCGGGGGCGGCGCGCGCGCGCGCGCGTGTGGTGTGCGTCGGAGGGCGGCGGCGGCGGCGGCGGCGGGGGTGTGTGGGGTCA CCCATACCCGGCCGTCGCCGGCAGTCGAGAGTGGACGGGAGCGGGGGGGCGGCGCGCGCGCGCGCGCGTGTGGTGTGCGTCGGAGGGCGGCGGCGGCGGCGGCGGCGGGGGTGTGGGGTTT CCCATACCCGGCCGTCGCCGGCAGTCGAGAGTGGACGGGAGCGGCGGGGCGGCGCGCGCGCGCGCGCGTGTGGTGTGCGTCGGAGGGCGGCGGCGGCGGCGGCGGCGGGGGTGTGGGGTC(2) CCCATACCCGGCCGTCGCCGGCAGTCGAGAGTGGACGGGAGCGGCGGGGGCGGCGCGCGCGCGCGCGTGTGGTGTGCGTCGGAGGGCGGCGGCGGCGGCGGCGGGGGTGTGGGGTCC CCCATACCCGGCCGTCGCCGGCAGTCGAGAGTGGACGGGAGCGGC

CCCATACCCGGCCGTCGCCGGCAGTCGAGAGTGGA

CCCATACCCGGCCGTCGCCGGCAGTCGAGAGTGG

CCCATACCCGGCCGTCGCCGGCAGTCGAGAGTG

CCCATACCCGGCCGTCGCCGGCAGTCGAGAGT(2)

CCCATACCCGGCCGTCGCCGGCAGTCGAGAGA(2)

CCCATACCCGGCCGTCGCCGGCAGTCGAGAGC

CCCATACCCGGCCGTCGCCGGCAGTCGAGAGG

CCCATACCCGGCCGTCGCCGGCAGTCGAGAA(3)

CCCATACCCGGCCGTCGCCGGCAGTCGAGAG

CCCATACCCGGCCGTCGCCGGCAGTCGAGA(17)

CCCATACCCGGCCGTCGCCGGCAGTCGAG(9)

CCCATACCCGGCCGTCGCCGGCAGTCGAA(11)

CCCATACCCGGCCGTCGCCGGCAGTCGAC

CCCATACCCGGCCGTCGCCGGCAGTCGA(3)

CCCATACCCGGCCGTCGCCGGCAGTCG

CCCATACCCGGCCGTCGCCGGCAGTCA

CCCATACCCGGCCGTCGCCGGCAGTC

CCCATACCCGGCCGTCGCCGGCAGA

CCCATACCCGGCCGTCGCCGGCAGG

g

h
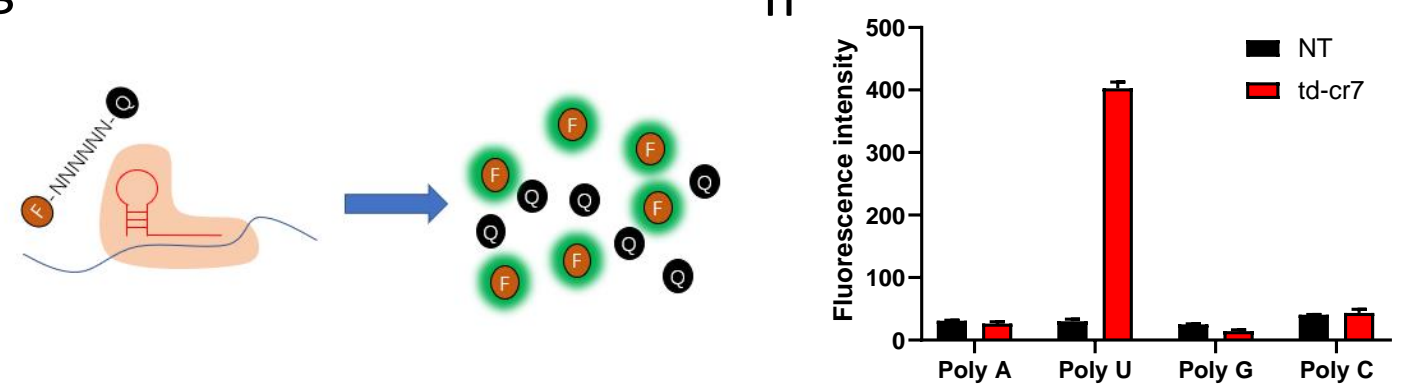

$\mathrm{F} 2+\mathrm{R} 2$ sequencing

....CCCCCGCCCCCCCCCCCACGCCTCCTCCCCTCCTCCCGCCCACGCCCCGCTCCCCGCCCCCGGAGCCCCGCGGACGCTACGCCGCGACGAGTAGGA Re (21)CCCCGCGGACGCTACGCCGCGACGAGTAGGA TCCCGCGGACGCTACGCCGCGACGAGTAGGA (3)CCCGCGGACGCTACGCCGCGACGAGTAGGA (2)CGGACGCTACGCCGCGACGAGTAGGA (4)ACGCTACGCCGCGACGAGTAGGA (8)CGCTACGCTGCGACGAGTAGGA (4)CTACGCTGCGACGAGTAGGA (6)TACGCTGCGACGAGTAGGA
GCGCTGCGACGAGTAGGA 
Figure S6

a

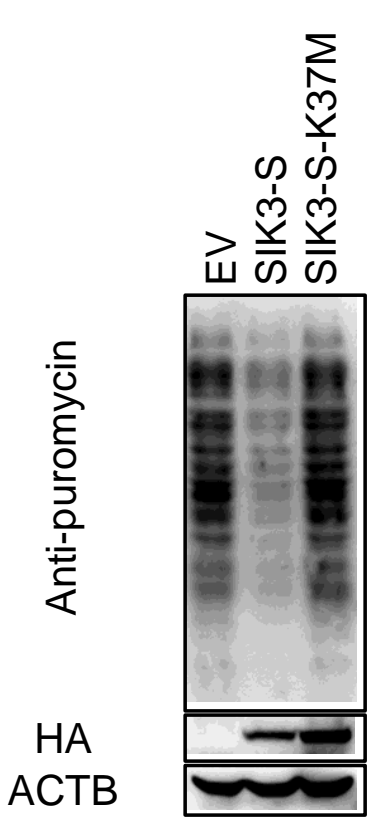

C

e

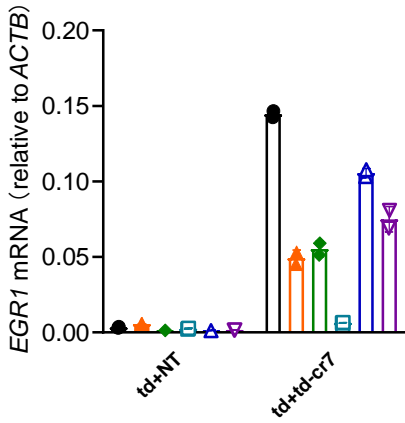

e

- DMSO

- SB203580 10uM

- SP600125 10uM

口 SB203580 10uM+SP600125 10uM

$\triangle$ U0126 10uM

$\nabla$ CCG-1423 10uM

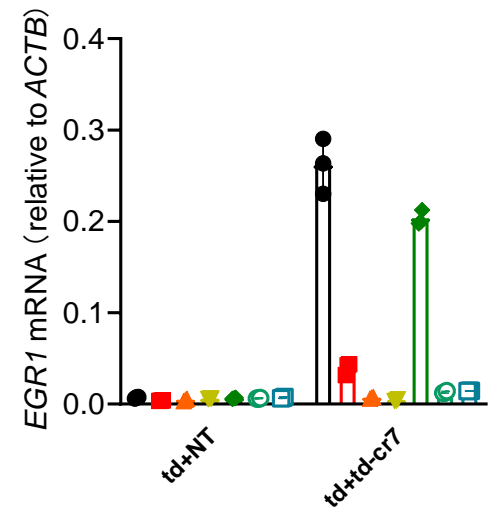

- DMSO

6P 0.1uM

- 6P 1uM

6P 10uM

- HY180 0.1uM

- HY180 1uM

HY180 10uM
$\mathrm{td}+\mathrm{NT}$

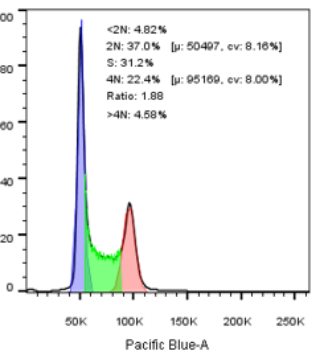

$\mathrm{K} 3+\mathrm{NT}$

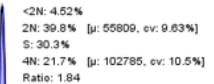

5: 30.3\$ [N: 102785, ov: 10.5\%]

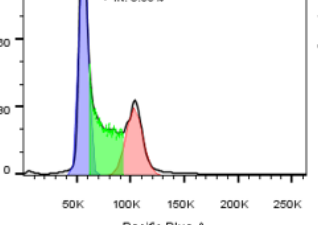

Pacific Blue-A
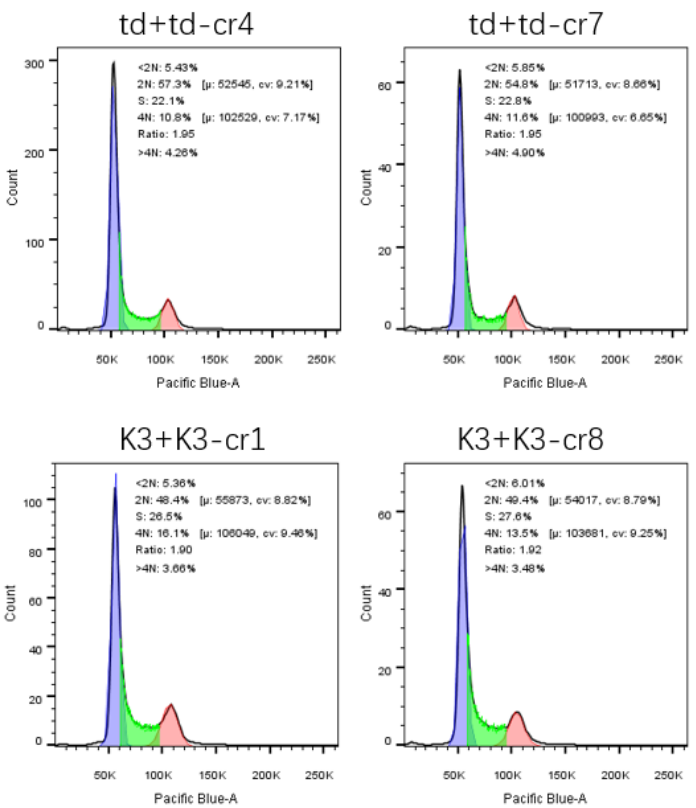

d
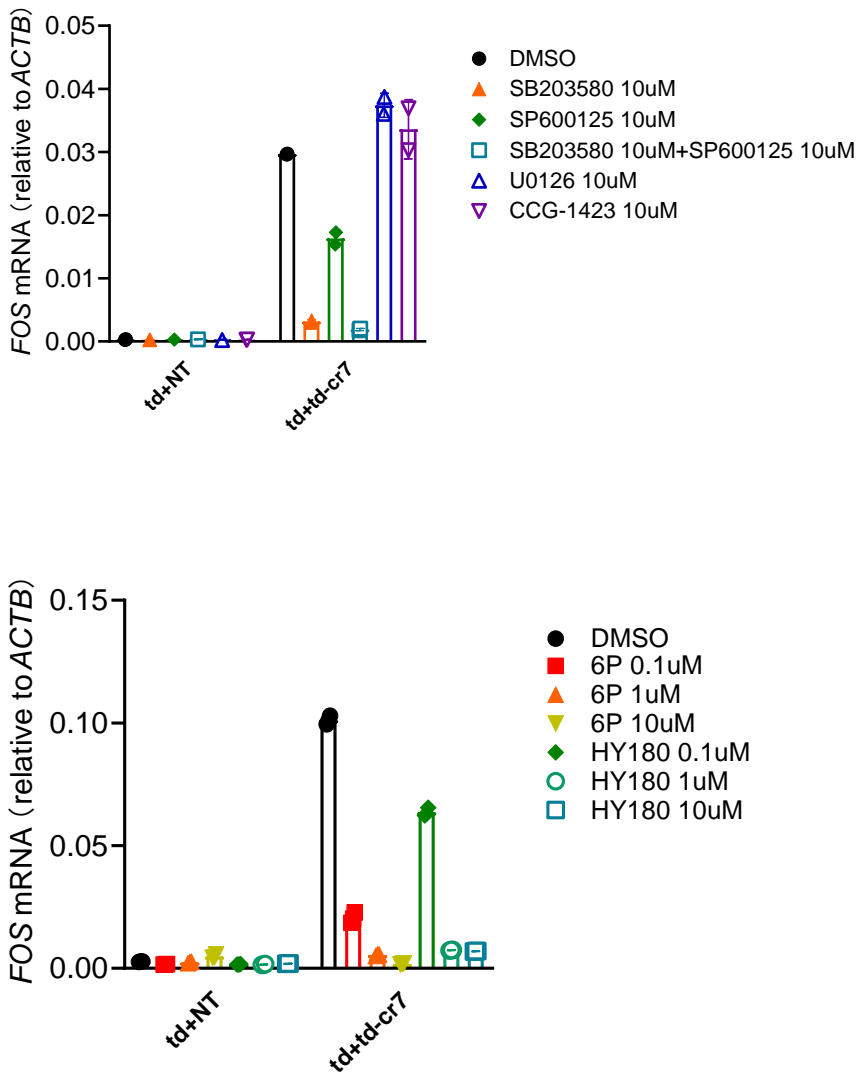

- DMSO

6P $0.1 \mathrm{uM}$

6P 1uM

6P $10 \mathrm{uM}$

- HY180 0.1uM

- HY180 1uM

HY180 10uM 


\section{Figure legend}

2 Figure 1. Mice died when knocking down Sik3-S in neurons using RfxCas13d.

3 a. Schematic illustration of RfxCas13d-mediated specifically knockdown of Sik3-S not Sik3-L

4 mRNA. Sik3-L: a transcript encoding a long isoform of SIK3.

5 b. RT-qPCR to measure the knockdown efficiency of Sik3-S crRNAs.

6 c. Transcriptome analysis in N2a cells $48 \mathrm{~h}$ post transfection of plasmids encoding RfxCas $13 \mathrm{~d}$ and

7 Sik3-S crRNAs or NT crRNA. $(\mathrm{n}=3)$

8 d. Workflow of knocking down Sik3-S in the adult mouse brain neurons.

9 e. Body weight change curve of ${ }^{\text {LSL }} \mathrm{RfxCas} 13 \mathrm{~d}$ mice after injection of AAV-PHP.eB carrying Sik3-S crRNAs or NT crRNA. $(\mathrm{n}=5)$

f. Survival curve of ${ }^{\text {LSL }}$ RfxCas13d mice after injection of AAV-PHP.eB carrying Sik3-S crRNAs or NT crRNA. $(n=5)$

g. Western blot to measure SIK-S expression level in brain lysates of ${ }^{\mathrm{LSL}} \mathrm{RfxCas} 13 \mathrm{~d}$ mice injected with AAV-PHP.eB carrying Sik3-S crRNAs or NT crRNA at 20 dpi.

Figure S1. Mice died when knocking down Sik3-S in neurons using RfxCas13d.

\section{Linked to Figure 1.}

a. Schematic illustration of alternative splicing process to generate $S i k 3-L$ and $S i k 3-S$ transcripts, and design of Sik3-S crRNAs.

b. Workflow of measuring the knockdown efficiency of crRNAs in N2a or HEK293T cells.

c. Schematic diagram of ${ }^{\text {LSL }}$ RfxCas13d mice. NLS: nuclear localization sequence; HA: hemagglutinin tag; CAG: CAG promoter; U6: U6 promoter; STOP: a tripartite transcriptional stop cassette.

d. Schematic illustration of AAV-PHP.eB plasmid used. hSYN: human synapsin 1 gene promoter.

Figure 2. RfxCas13d mediated lethality was not due to the loss of target gene function.

a. Western blot to measure SIK3-S expression level in brain lysates from Sik3-E5 ${ }^{\text {flox }}$ and WT mice at 21 dpi.

b. Survival curve of Sik3-E5 ${ }^{\text {flox }}$ and WT mice after injection of AAV-PHP.eB-hSYN-Cre. $(n=5)$

c. Body weight change curve of Sik3-E5 ${ }^{\text {flox }}$ and WT mice after injection of AAV-PHP.eB-hSYN-Cre. $(n=5)$

d-e. RT-qPCR to measure the knockdown efficiency of Mapt and Map2 crRNAs in N2a cells.

f. Western blot to measure the knockdown efficiency of Rbfox3 crRNAs by knocking down overexpressing NeuN in HEK293T cells using RfxCas13d.

g-i. Transcriptome analysis in N2a cells $48 \mathrm{~h}$ after transfection of plasmids encoding RfxCas13d and crRNAs. $(n=3)$

j-1. Body weight change curve of ${ }^{\mathrm{LSL}}$ RfxCas13d mice after infection of PHP.eB carrying Map2 crRNAs, Mapt crRNAs or Rbfox3 crRNAs. ( $\mathrm{n}=5)$

m-o. Survival curve of ${ }^{\text {LSL }}$ RfxCas13d mice after injection of AAV-PHP.eB carrying Map2 crRNAs, Mapt crRNAs or Rbfox3 crRNAs. (n=5) in brain lysates of ${ }^{\mathrm{LSL}} \mathrm{Rf}$ XCas13d mice after injection of AAV-PHP.eB carrying Map2 crRNAs, Mapt crRNAs or Rbfox3 crRNAs. 
44

45

46

47

48

49

50

51

52

53

54

55

56

57

58

59

60

61

62

63

64

65

66

67

68

69

70

71

72

73

74

75

76

77

78

79

80

81

82

83

84

85

Figure S2. RfxCas13d mediated lethality was not due to the loss of target gene function.

\section{Linked to Figure 2.}

a. Schematic illustration of knocking out Sik3 by AAV-PHP.eB-hSYN-Cre injection of Sik3-E ${ }^{\text {flox }}$ mice.

Figure 3. RfxCas13d mediated lethality was not caused by off-target effects.

a. RT-qPCR to measure the knockdown efficiency of tdTomato crRNAs in N2a cells stably expressing tdTomato (N2a-td).

b. Transcriptome analysis of N2a-td cells $48 \mathrm{~h}$ after transfection of plasmids encoding RfxCas $13 \mathrm{~d}$ and tdTomato crRNAs or NT crRNA. $(\mathrm{n}=3)$

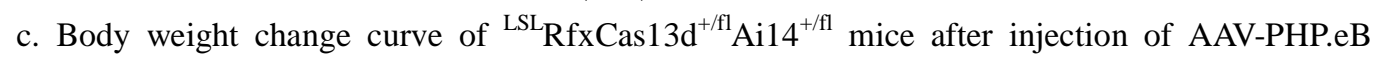
carrying tdTomato crRNAs or NT crRNA. $(n=4)$

d. Survival curve of ${ }^{\mathrm{LSL}} \mathrm{RfxCas} 13 \mathrm{~d}^{+/ f 1} \mathrm{Ai} 14^{+/ f l}$ mice after injection of AAV-PHP.eB carrying tdTomato crRNAs or NT crRNA. $(n=4)$

e. Western blot to measure tdTomato expression level in brain lysates of ${ }^{\mathrm{LSL}} \mathrm{RfxCas} 13 \mathrm{~d}^{+/ f 1} \mathrm{Ai} 14^{+/ f 1}$ mice injected with AAV-PHP.eB carrying tdTomato crRNAs or NT crRNA at $12 \mathrm{dpi}$.

f. Schematic of delivering AAV-PHP.eB carrying tdTomato crRNAs or NT crRNA into ${ }^{\mathrm{LSL}} \mathrm{RfxCas} 13 \mathrm{~d}^{+/ f 1} \mathrm{Ai} 14^{+/ f 1}$ and ${ }^{\mathrm{LSL}} \mathrm{RfxCas} 13 \mathrm{~d}$ mice, and the possible outcomes.

g. Body weight change curve of ${ }^{\mathrm{LSL}} \mathrm{Rfx}$ Cas $13 \mathrm{~d}$ and ${ }^{\mathrm{LSL}} \mathrm{RfxCas} 13 \mathrm{~d}^{+/ f 1} \mathrm{Ai} 14^{+/ f 1}$ mice after injection of AAV-PHP.eB carrying tdTomato crRNAs or NT crRNA. $(n=4)$

h. Survival curve of ${ }^{{ }^{L S L}} \mathrm{RfxCas} 13 \mathrm{~d}$ and ${ }^{\mathrm{LSL}} \mathrm{RfxCas} 13 \mathrm{~d}^{+/ f l} \mathrm{Ai} 14^{+/ f l}$ mice after injection of AAV-PHP.eB carrying tdTomato crRNAs or NT crRNA. $(n=4)$

Figure S3. RfxCas13d mediated lethality was not caused by off-target effects.

\section{Linked to Figure 3.}

a. Schematic diagram of Ai14 reporter mice

b. Schematic illustration of generating ${ }^{\mathrm{LSL}} \mathrm{Cas} 13 \mathrm{~d}^{+/ f 1} \mathrm{Ai} 14^{+/ f 1}$ mice by crossing ${ }^{\mathrm{LSL}} \mathrm{RfxCas} 13 \mathrm{~d}$ mice with Ai14 mice.

Figure 4. The collateral activity of RfxCas13d was determined by the abundance of target RNA in mammalian cells.

a. Western blot to measure the expression level of RfxCas13d and tdTomato $24 \mathrm{~h}$ after transfection plasmids encoding RfxCas13d, tdTomato and crRNAs into HEK293T cells. 3F-tdTomato means tdTomato with $3 x$ Flag tag at $\mathrm{N}$-terminal.

b. RT-qPCR to measure the RNA level of RfxCas13d in a. td represents tdTomato. EV represents empty vector.

c. Western blot to measure the expression level of RfxCas $13 \mathrm{~d}$ and tdTomato $24 \mathrm{~h}$ after transfection of plasmids encoding RfxCas13d, tdTomato and crRNAs into inducible-expressing tdTomato HEK293T cells. Cells were treated with different doxycycline concentration in advance.

d-f. RT-qPCR to measure the knockdown efficiency of ACTB, LDHB and YEHAE crRNAs in HEK293T cells. 
86

87

88

89

90

91

92

93

94

95

96

97

98

99

100

101

102

103

104

105

106

107

108

109

110

111

112

113

114

115

116

117

118

119

120

121

122

123

124

125

126

127

g-i. Western blot to measure the expression level of RfxCas13d/dRfxCas13d $24 \mathrm{~h}$ after transfection of plasmids encoding RfxCas13d/dRfxCas13d and crRNAs into HEK293T cells. cr-1/2/3/4: crRNA 1/2/3/4.

Figure S4. The collateral activity of RfxCas13d was determined by the abundance of target RNA in mammalian cells.

Linked to Figure 4.

a. RT-qPCR to measure the RNA level of EGFP after transfecting in vitro-synthesized EGFP crRNAs into U87 cells stably expressing LwaCas13a and EGFP.

b. RNA denaturing gel to measure the integrity of total RNA in a.

c. Western blot to measure the expression level of RfxCas13d and SIK3-S $24 \mathrm{~h}$ after transfection plasmids encoding RfxCas13d, SIK3-S and crRNAs into HEK293T cells.

d. RT-qPCR to measure the RNA level of RfxCas13d in c. K3 represents SIK3-S; EV represents empty vector.

e-f. Western blot to measure the expression level of LwaCas13a/PspCas13b and tdTomato $24 \mathrm{~h}$ after transfection of plasmids encoding LwaCas13a/PspCas13b, tdTomato and corresponding crRNAs into HEK293T cells. td-cr1: tdTomato crRNA 1; td-cr2: tdTomato crRNA 2.

g-j. RT-qPCR to measure the knockdown efficiency of GAS5, HNRNPAB, NCL and TPT1 crRNAs in HEK293T cells

k-n. Western blot to measure the expression level of RfxCas13d/dRfxCas13d $24 \mathrm{~h}$ after transfection of plasmids encoding RfxCas13d/dRfxCas13d and crRNAs into HEK293T cells. cr-1/2/3/4: crRNA 1/2/3/4.

Figure 5. The collateral activity of RfxCas13d cleaves 28s rRNA into two fragments, leading to translation attenuation and activation of ZAKo-JNK/p38-IEG pathway.

a. Total RNA of each sample was quantified by Agilent 2200 Bioanalyzer. td: tdTomato; td-cr4/7: tdTomato crRNA 4/7; K3-cr1/8: Sik3-S crRNA 1/8.

b. Schematic illustration of the cleavage site of RfxCas13d on human 28s rRNA.

c. SUnSET essay to measure the protein translation rate of HEK293T-RfxCas13d cells $24 \mathrm{~h}$ after transfection of plasmids encoding tdTomato/SIK3-S-K37M and corresponding crRNAs.

d. Statistical diagram of cell cycle distribution in Fig. S6b.

e. Schematic illustration of 509 common differentially expressed genes from four sets of comparisons.

f. Heatmap to demonstrate the expression level of IEGs in different groups. $\log _{2}$ (foldchange).

g. Transcription factor enrichment analysis using ChEA3. There are Top 15 transcription factors.

h. Western blot to measure the phosphorylation level of p38, JNK and ERK in HEK293T-RfxCas13d $24 \mathrm{~h}$ after transfection of plasmids encoding tdTomato and tdTomato crRNAs or NT crRNA. Red asterisk represents non-specific band.

i-j. RT-qPCR to measure the RNA level of EGR1 and FOS in HEK293T-RfxCas13d cells $24 \mathrm{~h}$ after transfection of plasmids encoding tdTomato and tdTomato crRNAs or NT crRNA.

k. Western blot to measure the expression level of ZAKa, ZAK $\beta$ and PKR in indicated cells. PKR-KO-1/2\#: two strain of PKR knockout HEK293T-RfxCas13d cells; ZAK-KO-1/2\#: two 
strain of ZAK knockout HEK293T-RfxCas13d cells; ZAK-KO-2\#-r-ZAKa: re-expression of ZAK $\alpha$ in ZAK-KO-2\#; ZAK-KO-2\#-r-ZAK $\beta$ : re-expression of ZAK $\beta$ in ZAK-KO-2\#

1. Western blot to measure the phosphorylation level of p38, JNK $24 \mathrm{~h}$ after transfection of plasmids encoding RfxCas13d and tdTomato crRNA 7 or NT crRNA into indicated cells. Red

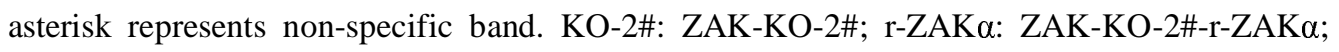
r-ZAK $\beta$ : ZAK-KO-2\#-r-ZAK $\beta$.

$\mathrm{m}$. Schematic illustration that the collateral activity of RfxCas13d cleaves $28 \mathrm{~s}$ rRNA into two fragments, leading to translation attenuation and activation of ZAK $\alpha-\mathrm{p} 38 / \mathrm{JNK}-\mathrm{IEGs}$ pathway.

Figure S5. The collateral activity of RfxCas13d cleaves 28 s rRNA into two fragments, leading to translation attenuation and activation of ZAK $\alpha-J N K / p 38$-IEG pathway.

\section{Linked to Figure 5.}

a. Workflow of experiments did in HEK293T-RfxCas13d cells.

b. Schematic illustration that the collateral activity of RfxCas13d cleaves its own mRNA and 28s RNA, thereby inhibiting its own protein expression.

c. Total RNA of each sample was quantified by Agilent 2200 Bioanalyzer. cr: crRNA.

d. Schematic illustration of oligonucleotide extension essay. Red represents oligonucleotide adaptor 1; Yellow represents oligonucleotide adaptor 2.

e. Gel picture of PCR products from d.

f. Sanger sequencing results. Ref: reference sequence. The numbers in brackets represent the number of identical sequencing results.

g. Schematic illustration of reconstitution of the collateral activity of RfxCas13d in vitro.

h. Statistical diagram of fluorescence intensity in $\mathrm{g}$.

i. Total RNA integrity analysis quantified by Agilent 2200 Bioanalyzer.

Figure S6. The collateral activity of RfxCas13d cleaves 28s rRNA into two fragments, leading to translation attenuation and activation of ZAK $\alpha$-JNK/p38-IEG pathway.

\section{Linked to Figure 5.}

a. SUnSET essay to measure the protein translation rate of HEK293T-RfxCas13d cells $24 \mathrm{~h}$ after transfection of plasmids encoding SIK3-S or SIK3-S-K37M.

b. Cell cycle analysis using FCAS.

c-d. RT-qPCR to measure the RNA level of EGR1 and FOS in HEK293T-RfxCas13d cells $24 \mathrm{~h}$ after transfection of plasmids encoding tdTomato and crRNAs. Inhibitors and transfection mix were added together. SB203580: p38 inhibitor; SP600125: JNK inhibitor; U0126: MEK1/2 inhibitor; CCG-1423: RhoA/C inhibitor.

e-f. RT-qPCR to measure the RNA level of EGR1 and FOS in HEK293T-RfxCas13d cells $24 \mathrm{~h}$ after transfection of plasmids encoding tdTomato and crRNAs into. ZAK inhibitors and transfection mix were added together. 6p and HY180: ZAK inhibitors. 
Table 1. The sequence of qPCR primers used in this study.

\begin{tabular}{|l|l|l|l|}
\hline Gene Name & Species & Forward primer & Reverse primer \\
\hline Sik3-S & Mouse & CAGAGCGACAGTGACCATCA & GCTGGATTCTGGTGAAAACCC \\
\hline Map2 & Mouse & ACCTTCCTCCATCCTCCCTC & TCCTGCTCTGCGAATTGGTT \\
\hline Mapt & Mouse & CCTCCTAGGCCTGACTCCTT & CTGGGGTGGAAGCGAAAGAT \\
\hline Actb & Mouse & TTACGGATGTCAACGTCACAGTTC & ACTATTGGCAACGAGCGGTTC \\
\hline TPT1 & Human & GGACTACCGTGAGGATGGTG & GGATTTCTTTCTTTTGCATCACATT \\
\hline LDHB & Human & GCCTTCTCTCTCCTGTGCAA & CCTCTTCTTCCGCAACTGGT \\
\hline NCL & Human & AAGCGTTGGAACTCACTGGT & TCTCGCATCTCGCTCTTTCT \\
\hline YWHAE & Human & GCAGAACTGGATACGCTGAG & ATTCTGCTCTTCACCGTCAC \\
\hline HNRNPAB & Human & TGTCAGTGGAAGCAAGTGTGA & CTGGTTCCAGTAGTTGCCGT \\
\hline GAS5 & Human & TATGGTGCTGGGTGCAGATG & CCATTAAGCTGGTCCAGGCA \\
\hline EGR1 & Human & ACCTGACCGCAGAGTCTTTT & CAAGGTGTTGCCACTGTTGG \\
\hline FOS & Human & TACACTCCAAGCGGAGACAG & TCCTTCAGCAGGTTGGCAAT \\
\hline GAPDH & Human & CAGCCTCAAGATCATCAGCA & TGTGGTCATGAGTCCTTCCA \\
\hline ACTB & Human & AAAGACCTGTACGCCAACAC & GTCATACTCCTGCTTGCTGAT \\
\hline RfxCas13d & & GACCCGTCCAGCAGGATATG & AGGCGGCGTTGGTAATGTAT \\
\hline tdTomato & & CCTGTTCCTGGGGCATGGCA & TCTTTGATGACGGCCATGTTGTTGT \\
\hline EGFP & & CGTAAACGGCCACAAGTTCA & CTTCATGTGGTCGGGGTAGC \\
\hline
\end{tabular}


Table 2. The sequence of crRNAs used in this study.

\begin{tabular}{|c|c|c|}
\hline Subtypes of Cas 13 & crRNA name & Sequence \\
\hline RfxCas13d & NT crRNA & TCACCAGAAGCGTACCATACTC \\
\hline RfxCas13d & Rbfox 3 crRNA 1 & CTGCATAGAATTCAGGCCCATA \\
\hline RfxCas13d & Rbfox3 crRNA 2 & GAAATGTATTATACACAGCACG \\
\hline $\operatorname{RfxCas} 13 \mathrm{~d}$ & Rbfox 3 crRNA 3 & GCCTCCATAAATCTCAGCACCA \\
\hline RfxCas13d & Rbfox 3 crRNA 4 & GAGCATATCTGTAAGCTGCATA \\
\hline RfxCas13d & Rbfox 3 crRNA 5 & AAATCCATCCTGATACACGACC \\
\hline $\operatorname{RfxCas} 13 \mathrm{~d}$ & Rbfox 3 crRNA 6 & GCAGCAACATTCAATGAGGCCA \\
\hline RfxCas13d & Sik3-S crRNA 1 & CAAGCACTGCCAGGTGCCACAT \\
\hline RfxCas13d & Sik3-S crRNA 2 & GCAGGGGAGCCTGCCCAAGGAC \\
\hline RfxCas13d & Sik3-S crRNA 3 & AATGGCGCTTATAGAAATGAAA \\
\hline RfxCas13d & Sik3-S crRNA 4 & GAAGAGGGAGGGAGGAGAGAAA \\
\hline RfxCas13d & Sik3-S crRNA 5 & TCACAGAAAATAAGAAAGACAA \\
\hline RfxCas13d & Sik3-S crRNA 6 & TCTCСААТСТССААСТССТТTТ \\
\hline RfxCas13d & Sik3-S crRNA 7 & GGAGATATTCATTCATTCATTC \\
\hline RfxCas13d & Sik3-S crRNA 8 & GTCGCTCTGTGAATCAGGCATC \\
\hline RfxCas13d & Map2 crRNA 1 & ACTCTCAATTTTCACACGTCCA \\
\hline RfxCas13d & Map2 crRNA 2 & TCAATCTTCACATTACCACCTC \\
\hline RfxCas13d & Map2 crRNA 3 & GAGCATTGTCAAGTGAGCCAAC \\
\hline RfxCas13d & Map2 crRNA 4 & CTTCGCCTGTTTAAAAGCACCA \\
\hline RfxCas13d & Map2 crRNA 5 & CCATGCAAAACAGAGCAGAGCG \\
\hline RfxCas13d & Map2 crRNA 6 & AAAGGAGAAGTATTCACAAGCC \\
\hline RfxCas13d & Mapt crRNA 1 & GTGATATTATCCAAGGAGCCAA \\
\hline RfxCas13d & Mapt crRNA 2 & CCACATCCCAGAATACCACCCC \\
\hline RfxCas13d & Mapt crRNA 3 & GCCAAGCATGAGAACAGGCAGA \\
\hline RfxCas13d & Mapt crRNA 4 & CTTAGATAAAAGAAAAGGCAGA \\
\hline RfxCas13d & Mapt crRNA 5 & AACTACAACGTAACAGGGCGAA \\
\hline RfxCas13d & Mapt crRNA 6 & ATAAAAGAAAAGGCAGAGGTCC \\
\hline RfxCas13d & tdTomato crRNA 1 & CCCTCGGAGCGCTCGTACTGTT \\
\hline RfxCas13d & tdTomato crRNA 2 & GGTGCCCTCGTAGGGGCGGCCC \\
\hline RfxCas13d & tdTomato crRNA 3 & GGGGGACAGGATGTCCCAGGCG \\
\hline RfxCas13d & tdTomato crRNA 4 & AGGAGTCCTGGGTCACGGTCAC \\
\hline RfxCas13d & tdTomato crRNA 5 & TCTTGGCCATGTAGATGGTCTT \\
\hline RfxCas13d & tdTomato crRNA 6 & CCAGACCGCCGTCCTCGAAGTT \\
\hline RfxCas13d & tdTomato crRNA 7 & GTCACCTTCAGCTTGGCGGTCT \\
\hline RfxCas13d & GAS5 5 crRNA 1 & CACACAGTGTAGTCAAGCCGAC \\
\hline RfxCas $13 d$ & GAS5 5 crRNA 2 & ATAAAAACGTTACCAGGAGCAG \\
\hline RfxCas13d & GAS5 5 crRNA 3 & AATTTATTAAAATTGGAGACAC \\
\hline RfxCas13d & GAS5 crRNA 4 & ATAAAAACGTTACCAGGAGCAG \\
\hline RfxCas 13d & HNRNPAB crRNA 1 & TTATTGTACAGTCAACGACCTC \\
\hline RfxCas13d & HNRNPAB crRNA 2 & CAACTCTGACTCTGACCTCCAC \\
\hline
\end{tabular}


bioRxiv preprint doi: https://doi.org/10.1101/2022.01.17.476700; this version posted January 18, 2022. The copyright holder for this preprint (which was not certified by peer review) is the author/funder. All rights reserved. No reuse allowed without permission.

\begin{tabular}{|c|c|c|}
\hline RfxCas13d & HNRNPAB crRNA 3 & CAAACAAAGCATGTGTGCGATC \\
\hline $\operatorname{RfxCas} 13 \mathrm{~d}$ & HNRNPAB crRNA 4 & CCTGGTAATAAAAATCAGCCCA \\
\hline RfxCas13d & TPT1 crRNA 1 & GTTCATGACAATATCGACACCA \\
\hline RfxCas $13 d$ & TPT1 crRNA 2 & AGTCCAATAGAGCAACCATGCC \\
\hline RfxCas $13 d$ & TPT1 crRNA 3 & ATTACCATTAACATGCAGCCTA \\
\hline $\operatorname{RfxCas} 13 \mathrm{~d}$ & TPT1 crRNA 4 & AGCTCAAGATGACATCAGTCCC \\
\hline $\operatorname{Rf} x \operatorname{Cas} 13 \mathrm{~d}$ & LDHB crRNA 1 & CCACAAGAGCAAGTTCATCAGC \\
\hline RfxCas13d & LDHB crRNA 2 & CCCAGAATGCTGATAGCACACG \\
\hline RfxCas $13 d$ & LDHB crRNA 3 & GGATTCAATAAGATCAGCCACA \\
\hline $\operatorname{Rf} x \operatorname{Cas} 13 \mathrm{~d}$ & LDHB crRNA 4 & CACACTTAATCCAATAGCCCAG \\
\hline RfxCas13d & NCL crRNA 1 & CCAACAAAGAGATTGAAAGCCG \\
\hline RfxCas13d & NCL crRNA 2 & TCCAACAAAGAGATTGAAAGCC \\
\hline RfxCas13d & NCL crRNA 3 & CAGGTAACAGTAAAAACCCCAG \\
\hline $\operatorname{RfxCas} 13 \mathrm{~d}$ & NCL crRNA 4 & GCAAACTCTATAAATGCATACC \\
\hline $\operatorname{RfxCas} 13 \mathrm{~d}$ & YWHAE crRNA 1 & CGGAAAAATTGAGAGCAAGACC \\
\hline RfxCas13d & YWHAE crRNA 2 & GTCATTGCAATATCACTAGCAG \\
\hline $\operatorname{RfxCas} 13 \mathrm{~d}$ & YWHAE crRNA 3 & AATAGAAAACCTTGGACTCGCC \\
\hline $\operatorname{RfxCas} 13 \mathrm{~d}$ & YWHAE crRNA 4 & CTACTTTCTTCATTGACTCCAC \\
\hline $\operatorname{RfxCas} 13 \mathrm{~d}$ & ACTB crRNA 1 & TGAAGCTGTAGCCGCGCTCGGT \\
\hline RfxCas13d & ACTB crRNA 2 & TGAACTTTGGGGGATGCTCGCT \\
\hline $\operatorname{Rf} x \operatorname{Cas} 13 \mathrm{~d}$ & ACTB crRNA 3 & CGTACAGGGATAGCACAGCCTG \\
\hline RfxCas13d & ACTB crRNA 4 & ATCTTGATCTTCATTGTGCTGG \\
\hline LwaCas13a & NT crRNA & CAGACTATGCGTCGACAAGCCAGGCATT \\
\hline LwaCas13a & tdTomato crRNA 1 & AAGCGCATGAACTCTTTGATGACCTCCT \\
\hline LwaCas13a & tdTomato crRNA 2 & TAGATGGTCTTGAACTCCACCAGGTAGT \\
\hline PspCas $13 b$ & NT crRNA & GTAATGCCTGGCTTGTCGACGCATAGTC \\
\hline PspCas $13 b$ & tdTomato crRNA 1 & AGGAGTCCTGGGTCACGGTCACCAGACC \\
\hline PspCas 13b & tdTomato crRNA 2 & GTCACCTTCAGCTTGGCGGTCTGGGTGC \\
\hline
\end{tabular}

